\title{
A FINITE-VOLUME SCHEME FOR THE MULTIDIMENSIONAL QUANTUM DRIFT-DIFFUSION MODEL FOR SEMICONDUCTORS
}

\author{
CLAIRE CHAINAIS-HILLAIRET, MARGUERITE GISCLON, AND ANSGAR JÜNGEL
}

\begin{abstract}
A finite-volume scheme for the stationary unipolar quantum drift-diffusion equations for semiconductors in several space dimensions is analyzed. The model consists of a fourth-order elliptic equation for the electron density, coupled to the Poisson equation for the electrostatic potential, with mixed Dirichlet-Neumann boundary conditions. The numerical scheme is based on a Scharfetter-Gummel type reformulation of the equations. The existence of a sequence of solutions to the discrete problem and its numerical convergence to a solution to the continuous model are shown. Moreover, some numerical examples in two space dimensions are presented.
\end{abstract}

AMS Classification. 35K35, 65N12, 76Y05.

Keywords. Quantum Bohm potential, density-gradient model, finite-volume method, discrete Sobolev inequality, existence of solutions, numerical convergence, quantum semiconductor devices.

\section{INTRODUCTION}

The performance of modern ultrasmall field-effect semiconductor devices is strongly influenced by quantum confinement transport close to the device gate or by direct tunneling through the channel potential barrier [26, 28]. In order to describe and numerically predict these effects, two main model approaches are possible, employing either a full quantum description of the charge transport or a macroscopic model including quantum corrections. The first approach may rely, for instance, on nonequilibrium Green's function models [9] or Wigner equations [16]. These models provide accurate physical results but they are computationally very costly, in particular in multidimensional simulations. The second approach includes quantum models derived from a Wigner-Boltzmann equation by using a moment method and suitable closure conditions [21]. There exists a hierarchy of macroscopic quantum models leading from quantum hydrodynamic equations [15, 18] to quantum energy-transport [10] and quantum drift-diffusion models [1]. The quantum drift-diffusion model has the advantage that well-established numerical methods developed for the classical drift-diffusion equations can be adopted. It is widely employed in the engineering community (see, e.g., [4, 35, 37]) and it is implemented in commercial device simulators like ISE and Silvaco. Moreover, it describes the correct behavior of quantum confinement and tunneling effects in MOSFET (metal-oxide semiconductor field-effect transistor) structures $[1,3]$. On the other hand, its numerical treatment is far from being trivial since it contains an equation with fourth-order derivatives, and a careful numerical analysis is needed.

The authors have been supported by the bilateral German-French DAAD-Procope Program. The last author acknowledges partial support from the Austrian Science Fund (FWF), grant P20214 and WK "Differential Equations", and from the German Science Foundation (DFG), grants JU 359/6 and JU 359/7. This research is part of the ESF program "Global and geometrical aspects of nonlinear partial differential equations (GLOBAL)". 
First numerical discretizations of the quantum drift-diffusion model were based on standard techniques, like finite differences [2] and standard finite elements [14]. High drain biases, however, may lead to large electric fields and large gradients of the electron density, which may give numerical difficulties in coarse grids when using standard techniques. These problems can be overcome by employing nonlinear schemes, such as the finite-difference nonlinear scheme of Ancona [2] or the Scharfetter-Gummel type discretization of Pinnau [32]. Numerical convergence results for these schemes can be found in $[32,33]$. For related results, we refer to [24]. Another direction is the use of finite-volume schemes [29]. However, no numerical analysis to show the numerical robustness of finite-volume schemes or multidimensional discretizations seems to be available in the literature. In this paper, we provide a finite-volume analysis for the multidimensional quantum drift-diffusion model for the first time.

The (scaled) stationary equations in a bounded domain $\Omega \subset \mathbb{R}^{d}(d \leq 3)$ read as

$$
\begin{aligned}
& \operatorname{div} J=0, \quad J=-\varepsilon^{2} n \nabla\left(\frac{\Delta \sqrt{n}}{\sqrt{n}}\right)+\nabla n-n \nabla V, \\
& \lambda^{2} \Delta V=n-C(x) .
\end{aligned}
$$

The variables are the electron density $n$, the electron current density $J$, and the electrostatic potential $V$. The physical parameters are the (scaled) Planck constant $\varepsilon>0$ and the Debye length $\lambda>0$. The function $C(x)$ represents the concentration of charged background ions (doping profile). The nonlocality of quantum mechanics is approximated by the fact that the equations of state do not only depend on the particle density but also on its gradient. Therefore, equations (1)-(2) are also called the density-gradient model.

Equation (1) contains fourth-order derivatives. It is convenient to reformulate (1) as a system of second-order equations which are numerically easier to treat than the original formulation. Introduce the quantum Fermi potential $F$ as the sum of the so-called Bohm potential $-\varepsilon^{2} \Delta \sqrt{n} / \sqrt{n}$, the thermodynamic potential $\log n$, and the electric potential $V$. Then we can write (1) as

$$
\operatorname{div}(n \nabla F)=0, \quad F=-\varepsilon^{2} \frac{\Delta \sqrt{n}}{\sqrt{n}}+\log n-V .
$$

The model equations are supplemented with physically motivated mixed boundary conditions. We assume that the boundary $\partial \Omega$ consists of contacts $\Gamma_{D}$ and insulating parts $\Gamma_{N}$. On the contacts, the electron density, the Fermi potential, and the electrostatic potential are prescribed, whereas the remaining boundary parts are insulating:

$$
\begin{array}{cc}
n=n_{D}, \quad F=F_{D}, \quad V=V_{D} & \text { on } \Gamma_{D}, \\
\nabla \sqrt{n} \cdot \nu=\nabla F \cdot \nu=\nabla V \cdot \nu=0 & \text { on } \Gamma_{N},
\end{array}
$$

where $\nu$ is the exterior unit normal vector to $\partial \Omega$. The boundary density $n_{D}$ is usually equal to the equilibrium density, and $V_{D}$ is the applied potential. The Dirichlet condition for $F$ is derived from the assumption that no quantum effects occur near the contacts, i.e., the quantum Bohm potential $\Delta \sqrt{n} / \sqrt{n}$ vanishes on $\Gamma_{D}$ [20], such that $F_{D}=\log n_{D}-V_{D}$. Noflux conditions for the electron current density and the electric field lead to homogeneous Neumann conditions on $\Gamma_{N}$ for $F$ and $V$, together with an additional homogeneous Neumann condition for $\sqrt{n}$.

The quantum drift-diffusion equations (1)-(2) were derived from the Wigner-BGK equation by a moment method and a Chapman-Enskog expansion [10]. The closure of the moment 
equations is realized by a quantum Maxwellian, which is the minimizer of the von Neumann quantum entropy subject to the constraint of a given particle density. The quantum drift-diffusion model can also be derived in the relaxation-time limit from the quantum hydrodynamic equations [22]. The existence of a weak solution to (2)-(3) was shown in [5]. Existence results for the one-dimensional transient equations can be found in [24, 25]. The multi-dimensional case (without pressure and electric field) was analyzed in [19, 23].

In this paper we analyze a finite-volume scheme applied to the stationary quantum driftdiffusion equations. Since the electron density might change by several orders of magnitude, we employ a Scharfetter-Gummel type formulation, similar as in [32]. Such a formulation was also utilized in the finite-volume scheme presented in [29] but with a different set of variables. Moreover, the paper [29] does not contain any numerical analysis.

Defining the total potential as the sum of the Bohm potential and the electric potential,

$$
G=-\varepsilon^{2} \frac{\Delta \sqrt{n}}{\sqrt{n}}-V
$$

and the Slotboom variable $u=e^{G} n$, we can formulate the current balance equation as

$$
\operatorname{div}\left(e^{-G} \nabla u\right)=0 .
$$

This expression corresponds to the exponential fitting formulation of the classical driftdiffusion equations [27]. Setting further $\rho=\sqrt{n}$, the system (2)-(3) is written in the new variables $(u, G, \rho, V)$ as the following system of second-order equations,

$$
\begin{aligned}
\operatorname{div}\left(e^{-G} \nabla u\right) & =0, \quad G=\log u-2 \log \rho, \\
-\varepsilon^{2} \Delta \rho & =\rho(\log u-2 \log \rho+V), \\
\lambda^{2} \Delta V & =\rho^{2}-C(x) \quad \text { in } \Omega,
\end{aligned}
$$

with the boundary conditions

$$
\begin{array}{cc}
u=u_{D}, \quad V=V_{D}, \quad \rho=\rho_{D} & \text { on } \Gamma_{D}, \\
\nabla u \cdot \nu=\nabla \rho \cdot \nu=\nabla V \cdot \nu=0 & \text { on } \Gamma_{N},
\end{array}
$$

where $u_{D}=n_{D} \exp \left(-V_{D}\right)$ and $\rho_{D}=\sqrt{n_{D}}$.

In the next section, we introduce the finite-volume scheme and present our main results. Furthermore, a discrete Sobolev inequality is proved. Section 3 is concerned with the proof of the existence of a solution to the numerical scheme. The proof is based on a fixed-point argument and the minimization of the discrete energy motivated by [5]. As a by-product, we show $L^{\infty}$ estimates independent of the discretization parameter using a discrete Stampacchia technique. A priori gradient estimates are proved in section 4. Furthermore, the convergence of (a subsequence of) approximate solutions to a solution to the continuous problem is shown. In Section 5, some numerical examples for simple two-dimensional ballistic and resonanttunneling diodes are given. Finally, we conclude in Section 6 .

\section{NUMERICAL SCHEME AND MAIN RESULTS}

In this section, we introduce some notations and our assumptions, present the finite-volume scheme for (4)-(6), and state our main results. Finally, we prove a discrete Sobolev inequality needed for the $L^{\infty}$ estimates below. 
2.1. Notations and assumptions. The domain $\Omega$ is an open bounded polygonal subset of $\mathbb{R}^{d}$ if $d=2$ (polyhedral if $d=3$ ). Its boundary satisfies

$$
\partial \Omega=\Gamma_{D} \cup \Gamma_{N} \in C^{0,1}, \Gamma_{D} \cap \Gamma_{N}=\emptyset, \Gamma_{N} \text { is open in } \partial \Omega, \text { meas }\left(\Gamma_{D}\right)>0 .
$$

In order to define the notion of weak solution for (4)-(8), we introduce the space

$$
H_{D}^{1}(\Omega)=\left\{\phi \in H^{1}(\Omega): \phi=0 \text { on } \Gamma_{D}\right\}
$$

(we note that $H_{D}^{1}(\Omega)=H_{0}^{1}(\Omega)$ when $\Gamma_{D}=\partial \Omega$ ). The boundary data are defined on the whole domain and satisfy

$$
u_{D}, \rho_{D}, V_{D} \in H^{1}(\Omega) \cap L^{\infty}(\Omega), \quad \inf _{\Gamma_{D}} \rho_{D}>0,
$$

and the doping profile satisfies

$$
C \in L^{2}(\Omega) \text {. }
$$

We say that $(u, \rho, V)$ is a weak solution to (4)-(8) if

$$
u \in u_{D}+H_{D}^{1}(\Omega), \quad \rho \in \rho_{D}+H_{D}^{1}(\Omega), \quad V \in V_{D}+H_{D}^{1}(\Omega)
$$

and if for all $\phi \in H_{D}^{1}(\Omega)$, it holds

$$
\begin{aligned}
\int_{\Omega} e^{-G} \nabla u \cdot \nabla \phi d x & =0, \quad G=\log (u)-2 \log (\rho) \text { in } \Omega, \\
\varepsilon^{2} \int_{\Omega} \nabla \rho \cdot \nabla \phi d x & =\int_{\Omega} \rho(\log u-2 \log \rho+V) \phi d x, \\
\lambda^{2} \int_{\Omega} \nabla V \cdot \nabla \phi d x & =-\int_{\Omega}\left(\rho^{2}-C(x)\right) \phi d x .
\end{aligned}
$$

An admissible mesh of $\Omega$ is given by a family $\mathcal{T}$ of control volumes (which are open and convex polygons in $\mathbb{R}^{2}$ and polyhedra in $\mathbb{R}^{3}$ ), a family $\mathcal{E}$ of edges in $\mathbb{R}^{2}$ (or faces in $\mathbb{R}^{3}$ ), and a family of points $\left(x_{K}\right)_{K \in \mathcal{T}}$ which satisfies Definition 5.1 in [12]. This definition implies in particular that the straight line between two neighboring centers of cells $\left(x_{K}, x_{L}\right)$ is orthogonal to the common edge $\sigma=K \mid L$. We assume that the triangulation is such that each edge (face) is contained in either $\Gamma_{D}$ or $\Gamma_{N}$. The size of the triangulation is defined by

$$
\operatorname{size}(\mathcal{T})=\max _{K \in \mathcal{T}} \operatorname{diam}(K)
$$

The set $\mathcal{E}$ is split into two subsets, the set $\mathcal{E}_{\text {int }}$ of interior edges and the set $\mathcal{E}_{\text {ext }}$ of boundary edges. Furthermore, we set $\mathcal{E}_{\text {ext }}=\mathcal{E}_{\text {ext }}^{D} \cup \mathcal{E}_{\text {ext }}^{N}$, where $\mathcal{E}_{\text {ext }}^{D}$ contains all Dirichlet boundary edges and $\mathcal{E}_{\text {ext }}^{N}$ all Neumann boundary edges. For a control volume $K \in \mathcal{T}$, we denote by $\mathcal{E}_{K}$ the set of its edges, $\mathcal{E}_{K \text {,int }}$ the set of its interior edges, $\mathcal{E}_{K \text {,ext }}^{D}$ the set of edges included in $\Gamma_{D}$, $\mathcal{E}_{K \text {,ext }}^{N}$ the set of edges included in $\Gamma_{N}$, and $\mathcal{E}_{K \text {,ext }}=\mathcal{E}_{K \text {,ext }}^{D} \cup \mathcal{E}_{K \text {,ext }}^{N}$. In the following, we denote by $\mathrm{d}$ the Euclidean distance in $\mathbb{R}^{d}$ and by $\mathrm{m}$ the measure in $\mathbb{R}^{d}$ or $\mathbb{R}^{d-1}$. For given $\sigma \in \mathcal{E}$, we define

$$
d_{\sigma}= \begin{cases}\mathrm{d}\left(x_{K}, x_{L}\right) & \text { if } \sigma \in \mathcal{E}_{\text {int }}, \sigma=K \mid L, \\ \mathrm{~d}\left(x_{K}, \sigma\right) & \text { if } \sigma \in \mathcal{E}_{\text {ext }}, \sigma \in \mathcal{E}_{K, \text { ext }},\end{cases}
$$

where $\mathrm{d}\left(x_{K}, \sigma\right)=\inf _{y \in \sigma} \mathrm{d}\left(x_{K}, y\right)$. We make the following regularity hypothesis on the mesh:

$$
\text { there exists } \zeta>0 \text { such that for all } K \in \mathcal{T}, \sigma \in \mathcal{E}_{K}: \mathrm{d}\left(x_{K}, \sigma\right) \geq \zeta d_{\sigma} \text {. }
$$

For all $\sigma \in \mathcal{E}$, we introduce the transmissibility coefficient $\tau_{\sigma}=\mathrm{m}(\sigma) / d_{\sigma}$. 
2.2. Finite-volume scheme and main results. The finite-volume scheme now writes, for all $K \in \mathcal{T}$, as

$$
\begin{aligned}
& \sum_{\substack{\sigma \in \mathcal{E}_{K, \text { int }}, \sigma=K \mid L}} \tau_{\sigma}\left(\rho_{K}-\rho_{L}\right)+\sum_{\sigma \in \mathcal{E}_{K, \text { ext }}^{D}} \tau_{\sigma}\left(\rho_{K}-\rho_{\sigma}^{D}\right)=\frac{\mathrm{m}(K)}{\varepsilon^{2}}\left(\log u_{K}-2 \log \rho_{K}+V_{K}\right) \rho_{K}, \\
& \sum_{\substack{\sigma \in \mathcal{E}_{K, \text { int }}, \sigma=K \mid L}} \tau_{\sigma}\left(V_{K}-V_{L}\right)+\sum_{\sigma \in \mathcal{E}_{K, \text { ext }}^{D}} \tau_{\sigma}\left(V_{K}-V_{\sigma}^{D}\right)=-\frac{\mathrm{m}(K)}{\lambda^{2}}\left(\rho_{K}^{2}-C_{K}\right), \\
& G_{K}=\log \left(u_{K}\right)-2 \log \left(\rho_{K}\right), \\
& \sum_{\substack{\sigma \in \mathcal{E}_{K, \text { int }}, \sigma=K \mid L}} \tau_{\sigma} e^{-\left(G_{K}+G_{L}\right) / 2}\left(u_{K}-u_{L}\right)+\sum_{\sigma \in \mathcal{E}_{K, \text { ext }}^{D}} \tau_{\sigma} e^{-G_{K}}\left(u_{K}-u_{\sigma}^{D}\right)=0,
\end{aligned}
$$

where the Dirichlet boundary conditions and the doping profile are discretized in the following way:

$$
\begin{array}{ll}
\rho_{\sigma}^{D}=\frac{1}{\mathrm{~m}(\sigma)} \int_{\sigma} \rho_{D}(s) d s, & u_{\sigma}^{D}=\frac{1}{\mathrm{~m}(\sigma)} \int_{\sigma} u_{D}(s) d s, \\
V_{\sigma}^{D}=\frac{1}{\mathrm{~m}(\sigma)} \int_{\sigma} V_{D}(s) d s, & C_{K}=\frac{1}{\mathrm{~m}(K)} \int_{K} C(x) d x
\end{array}
$$

for all $\sigma \subset \Gamma_{D}$ and $K \in \mathcal{T}$. Let $N$ be the number of control volumes in $\mathcal{T}$. The scheme (16)-(19) is a system of $4 N$ nonlinear equations in $4 N$ unknowns, $\left(\rho_{K}, V_{K}, G_{K}, u_{K}\right)_{K \in \mathcal{T}}$, or alternatively, in $3 N$ unknowns, $\left(\rho_{K}, V_{K}, u_{K}\right)_{K \in \mathcal{T}}$.

For a given mesh, the above scheme (if it admits a solution which will be proved below) provides some solution vectors of the form $\left(a_{K}\right)_{K \in \mathcal{T}}$, where $a=\rho, V, G, u$. We denote by $a$ the vector $\left(a_{K}\right)_{K \in \mathcal{T}}$ and by $a_{\mathcal{T}}$ the piecewise constant function, defined on $\Omega$, which equals $a_{K}$ on $K$. Let $X(\mathcal{T})$ be the set of functions from $\Omega$ to $\mathbb{R}$ which are constant on each control volume of the mesh. With this notation, it holds $a_{\mathcal{T}} \in X(\mathcal{T})$.

In Section 3, we will establish that there exists a solution to the finite-volume scheme. Our main result reads as follows.

Theorem 1. Let $\Omega$ be an open bounded polygonal or polyhedral subset of $\mathbb{R}^{d}(d \leq 3)$ satisfying (9) and let $\mathcal{T}$ be an admissible mesh satisfying (15). Furthermore, we assume that the boundary data and the doping profile satisfy (10) and (11). Then the finite-volume scheme (16)-(19) admits a solution $\left(\rho_{\mathcal{T}}, V_{\mathcal{T}}, u_{\mathcal{T}}\right) \in X(\mathcal{T})^{3}$ satisfying $\rho_{\mathcal{T}} \geq \rho>0$ in $\Omega$ for some $\rho$ independent of the triangulation.

Let $\left(\mathcal{T}_{h}\right)_{h>0}$ be a sequence of admissible meshes indexed by $h$, the size of the mesh. These meshes are supposed to satisfy (15) with $\zeta$ not depending on $h$. For $a=\rho, V, G$, $u$, we denote by $a_{h}=a_{\mathcal{T}_{h}} \in X\left(\mathcal{T}_{h}\right)$ an approximate solution given by the scheme on the mesh $\mathcal{T}_{h}$. In Section 4 , we prove the convergence of a sequence of approximate solution $\left(\rho_{h}, V_{h}, u_{h}\right)$ to a weak solution to (4)-(8) as $h \rightarrow 0$, as formulated in Theorem 2 below. For sake of simplicity, we assume that $\partial \Omega=\Gamma_{D}$, but the result still holds for mixed Dirichlet-Neumann boundary conditions.

Theorem 2. Let $\Omega$ be an open bounded polygonal or polyhedral subset of $\mathbb{R}^{d}(d \leq 3)$ satisfying (9) with $\partial \Omega=\Gamma_{D}$ and assume that the boundary data and the doping profile satisfy (10) and 
(11). Let $\left(\mathcal{T}_{h}\right)_{h>0}$ be a sequence of admissible meshes satisfying (15) and $\left(\rho_{h}, V_{h}, u_{h}\right)_{h>0}$ be a sequence of approximate solutions given by the scheme (16)-(19). Then there exists $(\widehat{\rho}, \widehat{V}, \widehat{u})$ such that, up to a subsequence, $\rho_{h} \rightarrow \widehat{\rho}, V_{h} \rightarrow \widehat{V}$ and $u_{h} \rightarrow \widehat{u}$ strongly in $L^{2}(\Omega)$ as $h \rightarrow 0$, and $(\widehat{\rho}, \widehat{V}, \widehat{u})$ is a weak solution to the system (4)-(8) satisfying $\widehat{\rho} \geq \underline{\rho}>0$ in $\Omega$.

2.3. A discrete Sobolev inequality. We denote by $\|\cdot\|_{q, \Omega}$ the usual norm on $L^{q}(\Omega)(1 \leq$ $q \leq \infty)$ and we write for $a_{\mathcal{T}} \in X(\mathcal{T})$

$$
\left\|a_{\mathcal{T}}\right\|_{q, \Omega}=\left(\sum_{K \in \mathcal{T}} \mathrm{m}(K) a_{K}^{q}\right)^{1 / q} \quad \text { if } q<\infty, \quad\left\|a_{\mathcal{T}}\right\|_{\infty, \Omega}=\sup _{K \in \mathcal{T}}\left|a_{K}\right| .
$$

Moreover, with a sequence $\left(a_{\sigma}\right)_{\sigma \in \mathcal{E}_{\text {ext }}^{D}}$, we define the discrete $H^{1}$-seminorm

$$
\left|a_{\mathcal{T}}\right|_{1, \mathcal{T}}=\left(\sum_{\substack{\sigma \in \mathcal{E}_{\text {int }}, \sigma=K \mid L}} \tau_{\sigma}\left(a_{K}-a_{L}\right)^{2}+\sum_{K \in \mathcal{T}} \sum_{\sigma \in \mathcal{E}_{K, \text { ext }}^{D}} \tau_{\sigma}\left(a_{K}-a_{\sigma}\right)^{2}\right)^{1 / 2} .
$$

By the sum over all $\sigma \in \mathcal{E}_{\text {int }}, \sigma=K \mid L$ we understand the sum of all interior edges $\sigma$ each of which is the common edge of some control volumes $K$ and $L$.

Lemma 3. Let $\Omega \subset \mathbb{R}^{d}(d \leq 3)$ be a polygonal or polyhedral domain satisfying (9) and let $\mathcal{T}$ be an admissible mesh satisfying (15). Furthermore, let $1 \leq q<\infty$ if $d=2$ and $1 \leq q \leq 6$ if $d=3$. Then there exists a constant $c_{S}>0$ depending only on $\Omega, \zeta, d$, and $q$ such that for all $a_{\mathcal{T}} \in X(\mathcal{T})$ and $\left(a_{\sigma}\right)_{\sigma \in \mathcal{E}_{\text {ext }}^{D}}$ satisfying $a_{\sigma}=0$ for all $\sigma \in \mathcal{E}_{\text {ext }}^{D}$,

$$
\left\|a_{\mathcal{T}}\right\|_{q, \Omega} \leq c_{S}\left|a_{\mathcal{T}}\right|_{1, \mathcal{T}}
$$

The discrete Sobolev inequality is shown in [8] in the special case $\partial \Omega=\Gamma_{D}$ and in [6] for $q=2$. We also refer to [13] for complementary results. The proof of Lemma 3, which is adapted from the proof in [6], is given here for completeness of the presentation.

Proof. We notice that $X(\mathcal{T})$ embeddes in the space $B V(\Omega)$ of functions of bounded variation and the latter space embeddes continuously into $L^{d /(d-1)}(\Omega)$. It can be shown (see [6, Lemma 3.2] for details) that there exists a constant $c(\Omega)>0$ only depending on $\Omega$ such that for all $v \in X(\mathcal{T})$ with $v_{\sigma}=0$ for all $\sigma \in \mathcal{E}_{\text {ext }}^{D}$,

$$
\left(\sum_{K \in \mathcal{T}} \mathrm{m}(K)\left|v_{K}\right|^{d /(d-1)}\right)^{(d-1) / d} \leq c(\Omega)\left(\sum_{\substack{\sigma \in \mathcal{E}_{\text {int }}, \sigma=K \mid L}} \mathrm{~m}(\sigma)\left|v_{K}-v_{L}\right|+\sum_{K \in \mathcal{T}} \sum_{\sigma \in \mathcal{E}_{K, \text { ext }}^{D}} \mathrm{~m}(\sigma)\left|v_{K}\right|\right) .
$$

We take $v=\left|a_{\mathcal{T}}\right|^{q(d-1) / d}$ and employ the fundamental inequality

$$
\left.|| a_{K}\right|^{q(d-1) / d}-\left|a_{L}\right|^{q(d-1) / d}\left|\leq \frac{q(d-1)}{d}\left(\left|a_{K}\right|^{q(d-1) / d-1}+\left|a_{L}\right|^{q(d-1) / d-1}\right)\right| a_{K}-a_{L} \mid
$$

for $q \geq d /(d-1)$. Then the Cauchy-Schwarz inequality and hypothesis (15) give

$$
\begin{aligned}
\left(\sum_{K \in \mathcal{T}} \mathrm{m}(K)\left|a_{K}\right|^{q}\right)^{(d-1) / d} \leq & \frac{2 c(\Omega) q(d-1)}{d}\left(\sum_{\substack{\sigma \in \mathcal{E}_{\text {int }}, \sigma=K \mid L}} \mathrm{~m}(\sigma)\left|a_{K}\right|^{q(d-1) / q-1}\left|a_{K}-a_{L}\right|\right. \\
& \left.+\sum_{K \in \mathcal{T}} \sum_{\sigma \in \mathcal{E}_{K, \text { ext }}^{D}} \mathrm{~m}(\sigma)\left|a_{K}\right|^{q(d-1) / d-1}\left|a_{K}\right|\right)
\end{aligned}
$$




$$
\begin{aligned}
& \leq \frac{2 c(\Omega) q(d-1)}{\sqrt{\zeta} d}\left|a_{\mathcal{T}}\right|_{1, \mathcal{T}}\left(\sum_{K \in \mathcal{T}} \sum_{\sigma \in \mathcal{E}_{K}} \mathrm{~m}(\sigma) \mathrm{d}\left(x_{K}, \sigma\right)\left|a_{K}\right|^{2 q(d-1) / d-2}\right)^{1 / 2} \\
& =\frac{2 c(\Omega) q(d-1)}{\sqrt{d \zeta}}\left|a_{\mathcal{T}}\right|_{1, \mathcal{T}}\left(\sum_{K \in \mathcal{T}} \mathrm{m}(K)\left|a_{K}\right|^{2 q(d-1) / d-2}\right)^{1 / 2},
\end{aligned}
$$

since $\sum_{\sigma \in \mathcal{E}_{K}} \mathrm{~m}(\sigma) \mathrm{d}\left(x_{K}, \sigma\right)=d \mathrm{~m}(K)$. Applying Hölder's inequality, we obtain

$$
\left(\sum_{K \in \mathcal{T}} \mathrm{m}(K)\left|a_{K}\right|^{2 q(d-1) / d-2}\right)^{1 / 2} \leq c^{\prime}(\Omega, q)\left(\sum_{K \in \mathcal{T}} \mathrm{m}(K)\left|a_{K}\right|^{q}\right)^{(d-1) / d-1 / q}
$$

and hence,

$$
\left\|a_{\mathcal{T}}\right\|_{q, \Omega}=\left(\sum_{K \in \mathcal{T}} \mathrm{m}(K)\left|a_{K}\right|^{q}\right)^{1 / q} \leq c_{S}\left|a_{\mathcal{T}}\right|_{1, \mathcal{T}}
$$

where $c_{S}=2 c(\Omega) c^{\prime}(\Omega, q) q(d-1) / \sqrt{d \zeta}$. The application of Hölder's inequality is possible only if $2 q(d-1) / d-2 \leq q$, which is true if $q<\infty$ and $d=2$ or if $q \leq 6$ and $d=3$.

\section{Existence OF A Discrete SOlution}

In this section, we prove Theorem 1. The proof is based on Brouwer's fixed-point theorem and discrete Stampacchia-type estimates. We assume that hypotheses (9), (10), (11), and (15) hold and we set

$$
\underline{u}=\inf _{\Gamma_{D}} u_{D}, \quad \bar{u}=\sup _{\Gamma_{D}} u_{D}, \quad \mathcal{U}=\left\{\left(u_{K}\right)_{K \in \mathcal{T}}: \underline{u} \leq u_{K} \leq \bar{u} \text { for all } K \in \mathcal{T}\right\} .
$$

The set $\mathcal{U}$ is convex and compact. The fixed-point mapping $T: \mathcal{U} \rightarrow \mathcal{U}, w \mapsto u$, is defined as follows:

(1) Compute $(\rho, V)=\left(\left(\rho_{K}\right)_{K \in \mathcal{T}},\left(V_{K}\right)_{K \in \mathcal{T}}\right)$ by solving scheme (16)-(17), where $u$ in (16) is replaced by $w$.

(2) Set $G_{K}=\log w_{K}-2 \log \rho_{K}$ for all $K \in \mathcal{T}$.

(3) Compute $u=\left(u_{K}\right)_{K \in \mathcal{T}}$ by solving scheme (19).

We need to prove that the mapping $T$ is well defined by establishing that each of the above steps admits a unique solution and that $T$ maps $\mathcal{U}$ into itself.

3.1. Existence and uniqueness of $(\rho, V)$. For given $w \in \mathcal{U}$, we show the existence and uniqueness for (16)-(17) (with $u$ replaced by $w$ ) by minimizing a discrete energy functional. To this end, we denote by $H$ the primitive of $\log$ which vanishes at the point $s=1(H(s)=$ $s \log (s)-s+1,0<s<\infty)$ and define the discrete energy functional

$$
\begin{aligned}
E(\rho, V)= & \varepsilon^{2} \sum_{\substack{\sigma \in \mathcal{E}_{\text {int }}, \sigma=K \mid L}} \tau_{\sigma}\left(\rho_{K}-\rho_{L}\right)^{2}+\varepsilon^{2} \sum_{K \in \mathcal{T}} \sum_{\sigma \in \mathcal{E}_{K, \text { ext }}^{D}} \tau_{\sigma}\left(\rho_{K}-\rho_{\sigma}^{D}\right)^{2} \\
& +\frac{\lambda^{2}}{2} \sum_{\substack{\sigma \in \mathcal{E}_{\text {int }}, \sigma=K \mid L}} \tau_{\sigma}\left(V_{K}-V_{L}\right)^{2}+\frac{\lambda^{2}}{2} \sum_{K \in \mathcal{T}} \sum_{\sigma \in \mathcal{E}_{K, \text { ext }}^{D}} \tau_{\sigma} V_{K}^{2} \\
& +\sum_{K \in \mathcal{T}} \mathrm{m}(K) H\left(\rho_{K}^{2}\right)-\sum_{K \in \mathcal{T}} \mathrm{m}(K) \rho_{K}^{2} \log w_{K} .
\end{aligned}
$$

The first two terms express the discrete quantum energy, the third and fourth term the discrete electric energy, and the last two terms are related to the discrete thermodynamic energy. For 
given $\rho=\left(\rho_{K}\right)_{K \in \mathcal{T}} \in \mathbb{R}^{N}$, scheme (17) admits a unique solution $V=\left(V_{K}\right)_{K \in \mathcal{T}} \in \mathbb{R}^{N}$, thus defining the mapping $\Phi: \mathbb{R}^{N} \rightarrow \mathbb{R}^{N}, \rho \mapsto V=\Phi(\rho)$ (recalling that $N$ is the number of control volumes). We can now define the functional $\eta:[0, \infty)^{N} \rightarrow \mathbb{R}, \eta(\rho)=E(\rho, \Phi(\rho))$.

Lemma 4. The functional $\eta$ admits a unique minimizer $\rho \in[0, \infty)^{N}$, and $(\rho, \Phi(\rho))$ is a solution to scheme (16)-(17).

Proof. The functional $\eta$ is well defined, continuous on the convex set $[0, \infty)^{N}$ and satisfies

$$
\eta(\rho) \geq \sum_{K \in \mathcal{T}} \mathrm{m}(K)\left(H\left(\rho_{K}^{2}\right)-\log (\bar{u}) \rho_{K}^{2}\right) .
$$

This implies that $\eta$ is bounded from below and weakly coercive $(\eta(\rho) \rightarrow \infty$ as $|\rho| \rightarrow \infty)$. Therefore, $\eta$ possesses a minimizer $\rho$ satisfying the Euler-Lagrange equations $\partial \eta / \partial \rho_{K}(\rho)=0$ for all $K \in \mathcal{T}$. The partial derivative of $\eta$ is given by

$$
\frac{\partial \eta}{\partial \rho_{K}}(\rho)=\frac{\partial E}{\partial \rho_{K}}(\rho, \Phi(\rho))+\sum_{K^{\prime} \in \mathcal{T}} \frac{\partial E}{\partial V_{K^{\prime}}}(\rho, \Phi(\rho)) \frac{\partial V_{K^{\prime}}}{\partial \rho_{K}}(\rho),
$$

where

$$
\begin{aligned}
\frac{\partial E}{\partial \rho_{K}}(\rho, \Phi(\rho))= & 2 \varepsilon^{2} \sum_{\substack{\sigma \in \mathcal{E}_{K, \text { int }}, \sigma=K \mid L}} \tau_{\sigma}\left(\rho_{K}-\rho_{L}\right)+2 \varepsilon^{2} \sum_{\sigma \in \mathcal{E}_{K, \text { ext }}^{D}} \tau_{\sigma}\left(\rho_{K}-\rho_{\sigma}^{D}\right) \\
& +2 \mathrm{~m}(K) \rho_{K} \log \left(\rho_{K}^{2}\right)-2 \mathrm{~m}(K) \rho_{K} \log w_{K}, \\
\frac{\partial E}{\partial V_{K^{\prime}}}(\rho, \Phi(\rho))= & \lambda^{2} \sum_{\substack{\sigma \in \mathcal{E}_{K^{\prime}}, \sigma=K^{\prime} \mid L}} \tau_{\sigma}\left(V_{K^{\prime}}-V_{L}\right)+\lambda^{2} \sum_{\sigma \in \mathcal{E}_{K^{\prime}, \mathrm{ext}}^{D}} \tau_{\sigma} V_{K^{\prime}} .
\end{aligned}
$$

Then the last term in (20) can be rewritten as

$$
\begin{aligned}
\sum_{K^{\prime} \in \mathcal{T}} \frac{\partial E}{\partial V_{K^{\prime}}}(\rho, \Phi(\rho)) \frac{\partial V_{K^{\prime}}}{\partial \rho_{K}}(\rho)= & \lambda^{2} \sum_{\substack{\sigma \in \mathcal{E}_{\text {int }}, \sigma=K^{\prime} \mid L}} \tau_{\sigma}\left(V_{K^{\prime}}-V_{L}\right)\left(\frac{\partial V_{K^{\prime}}}{\partial \rho_{K}}-\frac{\partial V_{L}}{\partial \rho_{K}}\right) \\
& +\lambda^{2} \sum_{K^{\prime} \in \mathcal{T}} \sum_{\sigma \in \mathcal{E}_{K^{\prime}, \mathrm{ext}}^{D}} \tau_{\sigma} V_{K^{\prime}} \frac{\partial V_{K^{\prime}}}{\partial \rho_{K}}
\end{aligned}
$$

In order to reformulate the first term on the right-hand side, we differentiate (17) (written for $K^{\prime}$ ) with respect to $\rho_{K}$ :

$$
\lambda^{2} \sum_{\substack{\sigma \in \mathcal{E}_{K^{\prime}}, \sigma=K^{\prime} \mid L}} \tau_{\sigma}\left(\frac{\partial V_{K^{\prime}}}{\partial \rho_{K}}-\frac{\partial V_{L}}{\partial \rho_{K}}\right)+\lambda^{2} \sum_{\sigma \in \mathcal{E}_{K^{\prime}, \text { ext }}^{D}} \tau_{\sigma} \frac{\partial V_{K^{\prime}}}{\partial \rho_{K}}=-2 \mathrm{~m}(K) \rho_{K} \delta_{K K^{\prime}} \quad \text { for all } K^{\prime} \in \mathcal{T},
$$

where $\delta_{K K^{\prime}}$ denotes the Kronecker symbol. Multiplying this equation by $V_{K^{\prime}}$ and summing over $K^{\prime} \in \mathcal{T}$ yields, after a discrete integration by parts,

$$
\lambda^{2} \sum_{\substack{\sigma \in \mathcal{E}_{\text {int }}, \sigma=K^{\prime} \mid L}} \tau_{\sigma}\left(\frac{\partial V_{K^{\prime}}}{\partial \rho_{K}}-\frac{\partial V_{L}}{\partial \rho_{K}}\right)\left(V_{K^{\prime}}-V_{L}\right)+\lambda^{2} \sum_{K^{\prime} \in \mathcal{T}} \sum_{\sigma \in \mathcal{E}_{K^{\prime}, \text { ext }}^{D}} \tau_{\sigma} \frac{\partial V_{K^{\prime}}}{\partial \rho_{K}} V_{K^{\prime}}=-2 \mathrm{~m}(K) \rho_{K} V_{K} .
$$


Inserting this expression into (21), the derivative (20) becomes

$$
\begin{aligned}
\frac{\partial \eta}{\partial \rho_{K}}(\rho)= & 2 \varepsilon^{2} \sum_{\substack{\sigma \in \mathcal{E}_{K, \text { int }}, \sigma=K \mid L}} \tau_{\sigma}\left(\rho_{K}-\rho_{L}\right)+2 \varepsilon^{2} \sum_{\sigma \in \mathcal{E}_{K, \text { ext }}^{D}} \tau_{\sigma}\left(\rho_{K}-\rho_{\sigma}^{D}\right) \\
& +2 \mathrm{~m}(K) \rho_{K} \log \left(\rho_{K}^{2}\right)-2 \mathrm{~m}(K) \rho_{K} \log w_{K}-2 \mathrm{~m}(K) \rho_{K} V_{K}, \quad K \in \mathcal{T} .
\end{aligned}
$$

This shows that the Euler-Lagrange equations are equivalent to scheme (16), and the minimizer $\rho$ is such that $(\rho, V=\Phi(\rho))$ solves (16)-(17).

We prove now that the minimizer is unique. The idea is to show the pseudo-convexity inequality

$$
\eta\left(\sqrt{\theta \rho_{1}^{2}+(1-\theta) \rho_{2}^{2}}\right)<\theta \eta\left(\rho_{1}\right)+(1-\theta) \eta\left(\rho_{2}\right)
$$

holds for all $0<\theta<1$ and $\rho_{1} \neq \rho_{2}$, from which the uniqueness follows immediately. This property has been first used in [30] for a quantum thermal equilibrium model. In order to prove (22), we split $\eta$ into three parts: the discrete quantum energy $\eta_{Q}$ (corresponding to the first two terms in $E(\rho, V)$ ), the discrete electric energy $\eta_{V}$ (corresponding to the third and fourth terms in $E(\rho, V)$ ), and the discrete thermodynamic-type energy $\eta_{H}$ (corresponding to the last two terms in $E(\rho, V))$. Since $H$ is strictly convex, the functional $\eta_{H}$ satisfies (22) with $\eta$ replaced by $\eta_{H}$. Furthermore, the mapping $\Phi$ is linear with respect to $\rho^{2}$ and $x \mapsto x^{2}$ is convex, implying that

$$
\eta_{V}\left(\sqrt{\theta \rho_{1}^{2}+(1-\theta) \rho_{2}^{2}}\right) \leq \theta \eta_{V}\left(\rho_{1}\right)+(1-\theta) \eta_{V}\left(\rho_{2}\right)
$$

for all $0 \leq \theta \leq 1$ and $\rho_{1}, \rho_{2}$. The functional $\eta_{Q}$ satisfies the same inequality (with $\eta_{V}$ replaced by $\left.\eta_{Q}\right)$ since the following elementary inequality holds for all $\left(x_{1}, x_{2}\right),\left(y_{1}, y_{2}\right) \in \mathbb{R}^{2}$ and $0 \leq \theta \leq 1$ :

$$
\left(\sqrt{\theta x_{1}^{2}+(1-\theta) x_{2}^{2}}-\sqrt{\theta y_{1}^{2}+(1-\theta) y_{2}^{2}}\right)^{2} \leq \theta\left(x_{1}-y_{1}\right)^{2}+(1-\theta)\left(x_{2}-y_{2}\right)^{2} .
$$

Indeed, we compute

$$
\begin{gathered}
\left(\sqrt{\theta x_{1}^{2}+(1-\theta) x_{2}^{2}}-\sqrt{\theta y_{1}^{2}+(1-\theta) y_{2}^{2}}\right)^{2}-\left(\theta\left(x_{1}-y_{1}\right)^{2}+(1-\theta)\left(x_{2}-y_{2}\right)^{2}\right) \\
=-2\left(\sqrt{\theta x_{1}^{2}+(1-\theta) x_{2}^{2}} \sqrt{\theta y_{1}^{2}+(1-\theta) y_{2}^{2}}-\left(\theta x_{1} y_{1}+(1-\theta) x_{2} y_{2}\right)\right)
\end{gathered}
$$

and an expansion of the right-hand side shows that it is nonpositive.

Remark 5. In Lemma 4, we have proved that the functional $\eta$ admits a unique minimizer $\rho$ and that $(\rho, V=\Phi(\rho))$ is a solution to the scheme (16)-(17). It implies that (16)-(17) has a solution but it does not necessarily imply that this solution is unique. In order to define the mapping $T$ without ambiguity, we precise in step (1) that $(\rho, V)$ is obtained by minimizing $\eta$.

3.2. $L^{\infty}$ estimates for $\rho$ and $V$. We show that $\rho$ and $V$ are bounded from above and below, using a Stampacchia technique [36] as suggested in [17].

Lemma 6. Let $(\rho, V)$ be a solution to (16)-(17) (guaranteed by Lemma 4). Then there exist constants $\underline{V}, \bar{V} \in \mathbb{R}$, and $\underline{\rho}, \bar{\rho}>0$ such that for all $K \in \mathcal{T}$,

$$
0<\underline{\rho} \leq \rho_{K} \leq \bar{\rho}, \quad \underline{V} \leq V_{K} \leq \bar{V}
$$


Proof. The strategy of the proof is as follows. First, we show that $V$ is bounded from above. This bound allows us to derive an upper bound for $\rho$, which provides a lower bound for $V$. Finally, the lower bound for $V$ implies the strict positivity of $\rho$.

First step: upper bound for $V$. We set $x^{+}=\max \{x, 0\}$ for $x \in \mathbb{R}$. Let $W \geq \sup _{\Gamma_{D}} V_{D}$. We multiply scheme $(17)$ by $\left(V_{K}-W\right)^{+}$and sum over $K \in \mathcal{T}$. By definition, we have $\left(V_{\sigma}^{D}-W\right)^{+}=0$ for all $\sigma \in \mathcal{E}_{\text {ext }}^{D}$. Then, after a discrete integration by parts,

$$
\begin{aligned}
\sum_{\substack{\sigma \in \mathcal{E}_{\text {int }}, \sigma=K \mid L}} \tau_{\sigma}\left(V_{K}-V_{L}\right)\left(\left(V_{K}-W\right)^{+}-\left(V_{L}-W\right)^{+}\right) & \\
& +\sum_{K \in \mathcal{T}} \sum_{\sigma \in \mathcal{E}_{K, \text { ext }}^{D}} \tau_{\sigma}\left(V_{K}-V_{\sigma}^{D}\right)\left(\left(V_{K}-W\right)^{+}-\left(V_{\sigma}^{D}-W\right)^{+}\right) \\
\leq & \frac{1}{\lambda^{2}} \sum_{K \in \mathcal{T}} \mathrm{m}(K)\left|C_{K}\right|\left(V_{K}-W\right)^{+} .
\end{aligned}
$$

Applying the following inequality on the left-hand side

$$
(x-y)\left(x^{+}-y^{+}\right) \geq\left(x^{+}-y^{+}\right)^{2} \text { for all }(x, y) \in \mathbb{R}^{2},
$$

and the Cauchy-Schwarz inequality on the right-hande side, we conclude that

$$
\begin{aligned}
\sum_{\substack{\sigma \in \mathcal{E}_{\text {int }}, \sigma=K \mid L}} \tau_{\sigma}\left(\left(V_{K}-W\right)^{+}-\left(V_{L}-W\right)^{+}\right)^{2} & +\sum_{K \in \mathcal{T}} \sum_{\sigma \in \mathcal{E}_{K, \text { ext }}^{D}} \tau_{\sigma}\left(\left(V_{K}-W\right)^{+}-\left(V_{\sigma}^{D}-W\right)^{+}\right)^{2} \\
\leq & \frac{1}{\lambda^{2}}\left(\sum_{K \in \mathcal{T}} \mathrm{m}(K) C_{K}^{2}\right)^{1 / 2}\left(\sum_{K \in \mathcal{T}} \mathrm{m}(K)\left(\left(V_{K}-W\right)^{+}\right)^{2}\right)^{1 / 2}
\end{aligned}
$$

which is rewritten as

$$
\left|\left(V_{\mathcal{T}}-W\right)^{+}\right|_{1, \mathcal{T}}^{2} \leq \frac{1}{\lambda^{2}}\|C\|_{2, \Omega}\left\|\left(V_{\mathcal{T}}-W\right)^{+}\right\|_{2, \Omega}
$$

We introduce the set $A_{W}=\left\{K \in \mathcal{T}: V_{K} \geq W\right\}$ and define $\mathrm{m}\left(A_{W}\right)=\sum_{K \in A_{W}} \mathrm{~m}(K)$. Applying Hölder's inequality, we find that

$$
\left\|\left(V_{\mathcal{T}}-W\right)^{+}\right\|_{2, \Omega} \leq\left\|\left(V_{\mathcal{T}}-W\right)^{+}\right\|_{q, \Omega} \mathrm{m}\left(A_{W}\right)^{1 / 2-1 / q}, \quad q>2 .
$$

Hence, by the discrete Sobolev inequality (Lemma 3),

$$
\left\|\left(V_{\mathcal{T}}-W\right)^{+}\right\|_{q, \Omega} \leq \mathrm{cm}\left(A_{W}\right)^{1 / 2-1 / q}, \quad 2<q \leq 6,
$$

where the constant $c>0$ depends on $\Omega, \zeta, d, q,\|C\|_{2, \Omega}$, and $\lambda$. Choosing $Z>W$ and observing that $A_{Z} \subset A_{W}$ and $\left(V_{K}-W\right)^{+} \geq Z-W$ for all $K \in A_{Z}$, we infer that

$$
\sum_{K \in \mathcal{T}} \mathrm{m}(K)\left(\left(V_{K}-W\right)^{+}\right)^{q} \geq \sum_{K \in A_{Z}} \mathrm{~m}(K)(Z-W)^{q},
$$

which is equivalent to

$$
\left\|\left(V_{\mathcal{T}}-W\right)^{+}\right\|_{q, \Omega} \geq \mathrm{m}\left(A_{Z}\right)^{1 / q}(Z-W)
$$


Thus, (23) and (24) imply that

$$
\mathrm{m}\left(A_{Z}\right) \leq \frac{c^{q}}{(Z-W)^{q}} \mathrm{~m}\left(A_{W}\right)^{q / 2-1}, \quad Z>W, 2<q \leq 6 .
$$

We can now apply the following lemma due to Stampacchia (see Lemma 4.1 in [36]).

Lemma 7. Let $\phi$ be a nonnegative and nondecreasing function, defined for all $W \geq W_{0}$, such that, if $Z>W>W_{0}$,

$$
\phi(Z) \leq \frac{c}{(Z-W)^{\alpha}} \phi(W)^{\beta},
$$

where $c \geq 0, \alpha>0$, and $\beta>1$. Then there exists $W^{*}>W_{0}$, depending only on $W_{0}, c, \alpha$, and $\beta$, such that $\phi\left(W^{*}\right)=0$.

We apply this lemma to the function $\phi(W)=\mathrm{m}\left(A_{W}\right)$ and $q>4$. Thus, there exists $\bar{V}$ such that $\mathrm{m}\left(A_{\bar{V}}\right)=0$ and hence, $V_{K} \leq \bar{V}$ for all $K \in \mathcal{T}$.

Second step: upper bound for $\rho$. We multiply (16) by $\left(\rho_{K}-M\right)^{+}$, where $M \geq \sup _{\Gamma_{D}} \rho_{D}$, and sum over $K \in \mathcal{T}$. Observing that, by definition, $\left(\rho_{\sigma}^{D}-M\right)^{+}=0$ for all $\sigma \in \mathcal{E}_{\text {ext }}^{D}$ and integrating by parts, we obtain

$$
\begin{aligned}
\sum_{\substack{\sigma \in \mathcal{E}_{\text {int }}, \sigma=K \mid L}} \tau_{\sigma}\left(\rho_{K}-\rho_{L}\right)\left(\left(\rho_{K}-M\right)^{+}-\left(\rho_{L}-M\right)^{+}\right) & \\
& +\sum_{K \in \mathcal{T}} \sum_{\sigma \in \mathcal{E}_{\mathrm{K}, \text { ext }}^{D}}\left(\rho_{K}-\rho_{\sigma}^{D}\right)\left(\left(\rho_{K}-M\right)^{+}-\left(\rho_{L}-M\right)^{+}\right) \\
= & \frac{1}{\varepsilon^{2}} \sum_{K \in \mathcal{T}} \mathrm{m}(K) \rho_{K}\left(\rho_{K}-M\right)^{+}\left(\log w_{K}-2 \log \rho_{K}+V_{K}\right) .
\end{aligned}
$$

The right-hand side is nonpositive if $M \geq M_{0}=\exp ((\log \bar{u}+\bar{V}) / 2)$. Hence, taking $\bar{\rho}=$ $\max \left\{\sup _{\Gamma_{D}} \rho_{D}, M_{0}\right\}$, we arrive at $\left|(\rho-\bar{\rho})^{+}\right|_{1, \mathcal{T}}^{2} \leq 0$, which implies that $\rho_{K} \leq \bar{\rho}$ for all $K \in \mathcal{T}$.

Third step: lower bound for $V$. This part is similar to the first step. Multiplying (17) by $-\left(W-V_{K}\right)^{+}$for $W \leq \inf _{\Gamma_{D}} V_{D}$, summing over $K \in \mathcal{T}$, and employing the upper bound for $\rho$ yields

$$
\left|\left(W-V_{\mathcal{T}}\right)^{+}\right|_{1, \mathcal{T}}^{2} \leq \frac{1}{\lambda^{2}}\|\bar{\rho}+C\|_{2, \Omega}\left\|\left(W-V_{\mathcal{T}}\right)^{+}\right\|_{2, \Omega},
$$

and the conclusion follows as in the first step.

Fourth step: lower bound for $\rho$. This is similar to the second step. Setting

$$
\underline{\rho}=\min \left\{\inf _{\Gamma_{D}} \rho_{D}, \exp \left(\frac{1}{2}(\log \underline{u}+\underline{V})\right)\right\}>0,
$$

we multiply $(16)$ by $\left(\underline{\rho}-\rho_{K}\right)^{+}$and sum over $K \in \mathcal{T}$. Then we proceed as in the second step.

3.3. Proof of Theorem 1. In section 3.1, we have proved the well-posedness of the first part of the definition of the fixed-point map $T$. In the second step, we define $G$ by $G_{K}=$ $\log w_{K}-2 \log \rho_{K}$ for $K \in \mathcal{T}$ (see (18)). The $L^{\infty}$ estimate for $\rho$ and the fact that $w \in \mathcal{U}$ imply $L^{\infty}$ bounds for $G$ :

$$
\underline{G}=\log \underline{u}-2 \log \bar{\rho} \leq G_{K} \leq \bar{G}=\log \bar{u}-2 \log \underline{\rho} \quad \text { for all } K \in \mathcal{T} .
$$

It remains to show that scheme (19) possesses a unique solution $u \in \mathcal{U}$. 
Actually, (19) is a system of linear equations. We consider the case $u_{\sigma}^{D}=0$ for all $\sigma \in \mathcal{E}_{K \text {,ext }}^{D}$, multiply (19) by $u_{K}$, and sum over $K \in \mathcal{T}$. Then

$$
\sum_{\substack{\sigma \in \mathcal{E}_{\text {int }}, \sigma=K \mid L}} \tau_{\sigma} e^{-\left(G_{K}+G_{L}\right) / 2}\left(u_{K}-u_{L}\right)^{2}+\sum_{K \in \mathcal{T}} \sum_{\sigma \in \mathcal{E}_{K, \text { ext }}^{D}} \tau_{\sigma} e^{-G_{K}} u_{K}^{2}=0 .
$$

Since $\mathrm{m}\left(\Gamma_{D}\right)>0$, this shows that $u_{K}=0$ for all $K \in \mathcal{T}$. Therefore, the matrix of the linear system is invertible, and the scheme admits a unique solution.

It is not difficult to derive $L^{\infty}$ bounds for $u$. Indeed, multiply (19) by $\left(u_{K}-\bar{u}\right)^{+}$, sum over $K \in \mathcal{T}$, and argue similarly as in the proof of Lemma 6 to obtain $\left|\left(u_{\mathcal{T}}-\bar{u}\right)^{+}\right|_{1, \mathcal{T}}^{2} \leq 0$ and hence, $u_{K} \leq \bar{u}$ for all $K \in \mathcal{T}$. In an analogous way, we find $u_{K} \geq \underline{u}$ for all $K \in \mathcal{T}$.

Thus, the mapping $T: \mathcal{U} \rightarrow \mathcal{U}, w \mapsto u$, introduced at the beginning of section 3 , is well defined and continuous. The Brouwer fixed-point theorem ensures the existence of $u \in \mathcal{U}$ such that $T(u)=u$. Let $(\rho, V)$ be the corresponding solution to (16)-(17) and define $G$ according to (18). Then $(\rho, V, G, u)$ is a solution to (16)-(19). Notice that we cannot conclude from Brouwer's theorem the uniqueness of solutions to the full system (16)-(19).

\section{NumericAl CONVERGENCE}

This section is devoted to the proof of Theorem 2. Given a solution $(\rho, V, G, u)$ to (16)(19) on a mesh of size $h$, we denote now by $\left(\rho_{\mathcal{T}}, V_{\mathcal{T}}, G_{\mathcal{T}}, u_{\mathcal{T}}\right) \in X(\mathcal{T})^{4}$ the corresponding approximate solution. First, we prove some discrete $H^{1}$ estimates satisfied by $\left(\rho_{\mathcal{T}}, V_{\mathcal{T}}, u_{\mathcal{T}}\right)$. Then, from a sequence of meshes $\left(\mathcal{T}_{h}\right)_{h>0}$ of size $h$, we can define a sequence of approximate solutions denoted by $\left(\rho_{h}, V_{h}, u_{h}\right)_{h>0}$. We show the compactness of the sequences $\left(\rho_{h}\right)_{h>0}$, $\left(V_{h}\right)_{h>0}$ and $\left(u_{h}\right)_{h>0}$ and weak compactness of some approximate gradients. These estimates allow us to pass to the limit in the numerical scheme.

\subsection{Discrete $H^{1}$ estimates.}

Lemma 8. Let $\Omega$ be an open bounded polygonal or polyhedral subset of $\mathbb{R}^{d}(d \leq 3)$ satisfying (9) and let $\mathcal{T}$ be an admissible mesh of size $h$ satisfying (15). Furthermore, we assume (10) and (11). Let $\left(\rho_{\mathcal{T}}, V_{\mathcal{T}}, G_{\mathcal{T}}, u_{\mathcal{T}}\right)$ be a solution to the finite-volume scheme (16)-(19). Then, there exists a constant $c>0$, not depending on $h$, such that

$$
\left|\rho_{\mathcal{T}}\right|_{1, \mathcal{T}} \leq c, \quad\left|V_{\mathcal{T}}\right|_{1, \mathcal{T}} \leq c, \quad\left|u_{\mathcal{T}}\right|_{1, \mathcal{T}} \leq c .
$$

Proof. We follow here the proof of Lemma 4.1 in [7]. First, we define the discrete functions $\rho_{\mathcal{T}}^{D}, V_{\mathcal{T}}^{D}$ and $u_{\mathcal{T}}^{D}$ by

$$
\rho_{\mathcal{T}}^{D}(x)=\rho_{K}^{D}, \quad V_{\mathcal{T}}^{D}(x)=V_{K}^{D}, \quad u_{\mathcal{T}}^{D}(x)=u_{K}^{D} \quad \forall x \in K,
$$

where $\rho_{K}^{D}$ (respectively $V_{K}^{D}, u_{K}^{D}$ ) is the $L^{2}$ projection of $\rho_{D}$ (respectively $V_{D}, u_{D}$ ) on $K$.

The scheme (16) rewrites

$$
\sum_{\substack{\sigma \in \mathcal{E}_{K, \text { int }}, \sigma=K \mid L}} \tau_{\sigma}\left(\rho_{K}-\rho_{L}\right)+\sum_{\sigma \in \mathcal{E}_{K, \text { ext }}^{D}} \tau_{\sigma}\left(\rho_{K}-\rho_{\sigma}^{D}\right)=\mathrm{m}(K) f_{K}
$$


with $f_{K}=\varepsilon^{-2}\left(\log u_{K}-2 \log \rho_{K}+V_{K}\right) \rho_{K}$. Multiplying (25) by $\rho_{K}-\rho_{K}^{D}$ and summing over $K \in \mathcal{T}$, we obtain

$$
\begin{aligned}
\sum_{K \in \mathcal{T}} \mathrm{m}(K) f_{K}\left(\rho_{K}-\rho_{K}^{D}\right)= & \sum_{\substack{\sigma \in \mathcal{E}_{\text {int }}, \sigma=K \mid L}} \tau_{\sigma}\left(\rho_{K}-\rho_{L}\right)\left(\left(\rho_{K}-\rho_{L}\right)-\left(\rho_{K}^{D}-\rho_{L}^{D}\right)\right) \\
& \quad+\sum_{K \in \mathcal{T}} \sum_{\sigma \in \mathcal{E}_{K, \text { ext }}^{D}} \tau_{\sigma}\left(\rho_{K}-\rho_{\sigma}^{D}\right)\left(\left(\rho_{K}-\rho_{\sigma}^{D}\right)-\left(\rho_{K}^{D}-\rho_{\sigma}^{D}\right)\right) \\
\geq & \frac{1}{2}\left(\left|\rho_{\mathcal{T}}\right|_{1, \mathcal{T}}^{2}-\left|\rho_{\mathcal{T}}^{D}\right|_{1, \mathcal{T}}^{2}\right) .
\end{aligned}
$$

Applying the Cauchy-Schwarz and Young inequality, we find that for $\alpha>0$,

$$
\sum_{K \in \mathcal{T}} \mathrm{m}(K) f_{K}\left(\rho_{K}-\rho_{K}^{D}\right) \leq \frac{1}{4 \alpha}\left\|f_{\mathcal{T}}\right\|_{2, \Omega}^{2}+\alpha\left\|\rho_{\mathcal{T}}-\rho_{\mathcal{T}}^{D}\right\|_{2, \Omega}^{2} .
$$

On the one hand, the $L^{\infty}$ estimates on $u, V$ and $\rho$ ensure that $\left\|f_{\mathcal{T}}\right\|_{2, \Omega}^{2}$ is bounded and, on the other hand, we can apply the discrete Sobolev inequality proven in Lemma 3, giving $\left\|\rho_{\mathcal{T}}-\rho_{\mathcal{T}}^{D}\right\|_{2, \Omega}^{2} \leq c_{S}^{2}\left(\left|\rho_{\mathcal{T}}\right|_{1, \mathcal{T}}^{2}+\left|\rho_{\mathcal{T}}^{D}\right|_{1, \mathcal{T}}^{2}\right)$. Choosing, for instance, $\alpha=1 /\left(4 c_{S}^{2}\right)$, we arrive at the desired estimate $\left|\rho_{\mathcal{T}}\right|_{1, \mathcal{T}} \leq c$.

The proof of the estimate $\left|V_{\mathcal{T}}\right|_{1, \mathcal{T}} \leq c$ is similar as the scheme (17) also rewrites under the form (25) with $V$ instead of $\rho$ and $f_{K}=-\lambda^{-2}\left(\rho_{K}^{2}-C_{K}\right)$. Finally, multiplying the scheme (18) by $u_{K}-u_{K}^{D}$, summing over $K \in \mathcal{T}$, and using the upper bound on $G$, we directly obtain $\left|u_{\mathcal{T}}\right|_{1, \mathcal{T}} \leq\left|u_{\mathcal{T}}^{D}\right|_{1, \mathcal{T}}$.

4.2. Compactness properties. In this section, we recall that the discrete $H^{1}$ estimates satisfied by a sequence of approximate solutions imply the strong convergence in $L^{2}$ of a subsequence of approximate solutions and the weak convergence of a sequence of approximate gradients.

Let us first define the approximate gradients. For each edge (face if $d=3$ ) of the mesh $\sigma$, we define the diamond $D_{\sigma}$ (see Figure 1 ) by

$$
\begin{aligned}
D_{\sigma}= & \left\{t x_{K}+(1-t) y, t \in[0,1], y \in \sigma\right\} \quad \text { if } \sigma \subset \partial \Omega, \sigma \in \mathcal{E}_{K, \text { ext }}, \\
D_{\sigma}= & \left\{t x_{K}+(1-t) y, t \in[0,1], y \in \sigma\right\} \\
& \cup\left\{t x_{L}+(1-t) y, t \in[0,1], y \in \sigma\right\} \quad \text { if } \sigma \in \mathcal{E}_{\text {int }}, \sigma=K \mid L .
\end{aligned}
$$

For an edge $\sigma \in \mathcal{E}_{K}$, we denote by $\nu_{K, \sigma}$ the normal to $\sigma$ outwards $K$.

Let $a_{\mathcal{T}} \in X(\mathcal{T})$ and $\left(a_{\sigma}\right)_{\sigma \in \mathcal{E}_{\text {ext }}^{D}}$ be some boundary values. As in [7] and [11], we define the approximate gradient $\nabla^{\mathcal{T}} a_{\mathcal{T}}(x)$ for $x \in D_{\sigma}$ by

$$
\nabla^{\mathcal{T}} a_{\mathcal{T}}(x)=\left\{\begin{array}{cl}
\frac{\mathrm{m}(\sigma)}{\mathrm{m}\left(D_{\sigma}\right)}\left(a_{L}-a_{K}\right) \nu_{K, \sigma} & \text { if } \sigma \in \mathcal{E}_{\mathrm{int}}, \sigma=K \mid L, \\
\frac{\mathrm{m}(\sigma)}{\mathrm{m}\left(D_{\sigma}\right)}\left(a_{\sigma}-a_{K}\right) \nu_{K, \sigma} & \text { if } \sigma \in \mathcal{E}_{\mathrm{ext}}^{D}, \\
0 & \text { if } \sigma \in \mathcal{E}_{\mathrm{ext}}^{N} .
\end{array}\right.
$$

In the sequel, we will write $\nabla^{h}=\nabla^{\mathcal{T}_{h}}$. Let us consider a sequence of admissible discretizations $\left(\mathcal{T}_{h}\right)_{h>0}$ of an open bounded polygonal subset $\Omega \subset \mathbb{R}^{d}$ and a sequence of functions $\left(a_{h}\right)_{h>0}$ with $a_{h} \in X\left(\mathcal{T}_{h}\right)$. We recall the convergence results established in [12] (for the functions) and [11] (for the gradients): if there exists $K>0$ such that $\left|a_{h}\right|_{1, \mathcal{T}_{h}} \leq K$ (we consider here the $H^{1}$ 


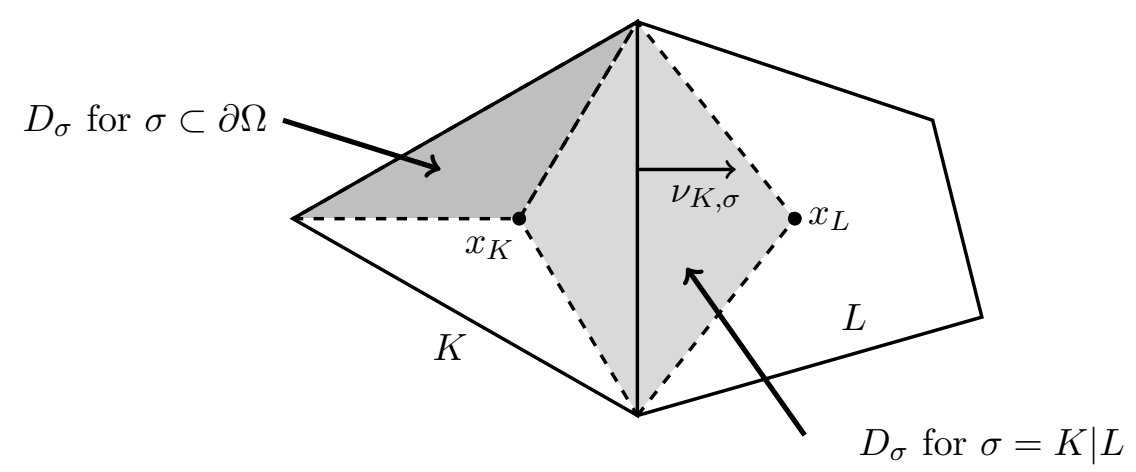

Figure 1. Definition of the diamond $D_{\sigma}$ associated to an edge $\sigma(d=2)$

seminorm with $\Gamma_{D}=\partial \Omega$ and $a_{\sigma}=0$ for $\left.\sigma \in \mathcal{E}_{\text {ext }}\right)$ for all $h>0$, then there exists $\widehat{a} \in H_{0}^{1}(\Omega)$ such that, up to a subsequence, $\left(a_{h}\right)$ converges to $\widehat{a}$ in $L^{2}(\Omega)$ and $\left(\nabla^{h} a_{h}\right)$ converges weakly to $\nabla \widehat{a}$ in $L^{2}(\Omega)^{d}$ as $h \rightarrow 0$. Due to the discrete $H^{1}$ estimates on $\rho_{\mathcal{T}}, V_{\mathcal{T}}$ and $u_{\mathcal{T}}$, we conclude the following convergence result.

Lemma 9. Let $\Omega$ be an open bounded polygonal or polyhedral subset of $\mathbb{R}^{d}(d \leq 3)$ satisfying (9) with $\partial \Omega=\Gamma_{D}$. Let $\left(\mathcal{T}_{h}\right)_{h>0}$ be a sequence of admissible meshes satisfying (15) and $\left(\rho_{h}, V_{h}, u_{h}\right)_{h>0}$ be a sequence of approximate solutions given by the scheme (16)-(19). Then there exists $(\widehat{\rho}, \widehat{V}, \widehat{u})$ such that $\widehat{\rho}-\rho^{D} \in H_{0}^{1}(\Omega), \widehat{V}-V^{D} \in H_{0}^{1}(\Omega), \widehat{u}-u^{D} \in H_{0}^{1}(\Omega)$ and, up to a subsequence, $\rho_{h} \rightarrow \widehat{\rho}, V_{h} \rightarrow \widehat{V}, u_{h} \rightarrow \widehat{u}$ strongly in $L^{2}(\Omega)$, as $h \rightarrow 0$, and $\nabla^{h} \rho_{h} \rightarrow \nabla \widehat{\rho}$, $\nabla^{h} V_{h} \rightarrow \nabla \widehat{V}, \nabla^{h} u_{h} \rightarrow \nabla \widehat{u}$ weakly in $L^{2}(\Omega)^{d}$.

4.3. Proof of Theorem 2. To conclude the proof of Theorem 2, it remains to prove that $(\widehat{\rho}, \widehat{V}, \widehat{u})$ obtained in Lemma 9 is a weak solution to (4)-(8). Therefore, we prove that the sequence of approximate solutions satisfies the weak formulation (12)-(14) with some error terms.

Lemma 10. Let $\Omega$ be an open bounded polygonal or polyhedral subset of $\mathbb{R}^{d}(d \leq 3)$ satisfying (9) with $\partial \Omega=\Gamma_{D}$. Let $\left(\mathcal{T}_{h}\right)_{h>0}$ be a sequence of admissible meshes satisfying (15) and $\left(\rho_{h}, V_{h}, u_{h}\right)_{h>0}$ be a sequence of approximate solutions given by the scheme (16)-(19). For all $\phi \in C_{c}^{\infty}(\Omega)$, it holds, for $h>0$ small enough, $G_{h}=\log \left(u_{h}\right)-2 \log \left(\rho_{h}\right)$ in $\Omega$ and

$$
\begin{aligned}
\left|\int_{\Omega} e^{-G_{h}} \nabla^{h} u_{h} \cdot \nabla \phi d x\right| & \leq K_{\phi} h, \\
\left|\varepsilon^{2} \int_{\Omega} \nabla^{h} \rho_{h} \cdot \nabla \phi d x-\int_{\Omega} \rho_{h}\left(\log u_{h}-2 \log \rho_{h}+V_{h}\right) \phi d x\right| & \leq K_{\phi} h, \\
\left|\lambda^{2} \int_{\Omega} \nabla^{h} V^{h} \cdot \nabla \phi+\int_{\Omega}\left(\rho_{h}^{2}-C(x)\right) \phi d x\right| & \leq K_{\phi} h,
\end{aligned}
$$

where $K_{\phi}>0$ does not depend on $h$.

Proof. We focus here on the proof of (28) as the proofs of (27) and (29) are similar.

Let $\phi \in C_{c}^{\infty}(\Omega)$. We suppose that $h=\operatorname{size}(\mathcal{T})$ is small enough such that $\operatorname{supp} \phi \subset\{x \in \Omega$ : $\mathrm{d}(x, \partial \Omega)>h\}$. Multiplying (16) by $\phi_{K}=\phi\left(x_{K}\right)$ and summing over $K \in \mathcal{T}$, we obtain the 
equation $\varepsilon^{2} A-B=0$, where

$$
A=\sum_{\substack{\sigma \in \mathcal{E}_{\text {int }}, \sigma=K \mid L}} \tau_{\sigma}\left(\rho_{K}-\rho_{L}\right)\left(\phi_{K}-\phi_{L}\right) \quad \text { and } \quad B=\sum_{K \in \mathcal{T}} \mathrm{m}(K)\left(\log u_{K}-2 \log \rho_{K}+V_{K}\right) \rho_{K} \phi_{K} .
$$

Since

$$
\begin{aligned}
\int_{\Omega} \nabla^{h} \rho_{h} \cdot \nabla \phi d x & =\sum_{\substack{\sigma \in \mathcal{E}_{\text {int }}, \sigma=K \mid L}} \int_{D_{\sigma}} \frac{\mathrm{m}(\sigma)}{\mathrm{m}\left(D_{\sigma}\right)}\left(\rho_{L}-\rho_{K}\right) \nabla \phi \cdot \nu_{K, \sigma} d x, \\
\int_{\Omega}\left(\log u_{h}-2 \log \rho_{h}+V_{h}\right) \rho_{h} \phi d x & =\sum_{K \in \mathcal{T}} \mathrm{m}(K)\left(\log u_{K}-2 \log \rho_{K}+V_{K}\right) \rho_{K} \frac{1}{\mathrm{~m}(K)} \int_{K} \phi d x,
\end{aligned}
$$

we have

$$
\begin{gathered}
A-\int_{\Omega} \nabla^{h} \rho_{h} \cdot \nabla \phi d x=\sum_{\substack{\sigma \in \mathcal{E}_{\text {int }}, \sigma=K \mid L}} \frac{\mathrm{m}(\sigma)}{\mathrm{m}\left(D_{\sigma}\right)}\left(\rho_{L}-\rho_{K}\right) \int_{D_{\sigma}}\left(\frac{\phi_{L}-\phi_{K}}{\mathrm{~d}_{\sigma}}-\nabla \phi \cdot \nu_{K, \sigma}\right) d x, \\
B-\int_{\Omega}\left(\log u_{h}-2 \log \rho_{h}+V_{h}\right) \rho_{h} \phi d x \\
=\sum_{K \in \mathcal{T}} \mathrm{m}(K)\left(\log u_{K}-2 \log \rho_{K}+V_{K}\right) \rho_{K}\left(\phi_{K}-\frac{1}{\mathrm{~m}(K)} \int_{K} \phi d x\right) .
\end{gathered}
$$

Observing that $\phi \in C_{c}^{\infty}(\Omega)$ and $\operatorname{size}(\mathcal{T})=h$, there exists $k_{\phi}>0$ depending only on $\phi$ such that, for all $K \in \mathcal{T}$ and $\sigma=K \mid L$,

$$
\left|\phi_{K}-\frac{1}{\mathrm{~m}(K)} \int_{K} \phi d x\right| \leq k_{\phi} h \quad \text { and } \quad\left|\frac{\phi_{L}-\phi_{K}}{\mathrm{~d}_{\sigma}}-\nabla \phi \cdot \nu_{K, \sigma}\right| \leq k_{\phi} h .
$$

Therefore, employing the Cauchy-Schwarz inequality and the discrete $H^{1}$ estimate for $\rho_{h}$, we find that

$$
\begin{aligned}
\left|A-\int_{\Omega} \nabla^{h} \rho_{h} \cdot \nabla \phi d x\right| & \leq \operatorname{ch} \sum_{\substack{\sigma \in \mathcal{E}_{\text {int }}, \sigma=K \mid L}} \mathrm{~m}(\sigma)\left|\rho_{L}-\rho_{K}\right| \\
& \leq \operatorname{ch}\left(\sum_{\substack{\sigma \in \mathcal{E}_{\text {int }}, \sigma=K \mid L}} \tau_{\sigma}\left|\rho_{L}-\rho_{K}\right|^{2}\right)^{1 / 2}\left(\sum_{\substack{\sigma \in \mathcal{E}_{\text {int }}, \sigma=K \mid L}} \mathrm{~m}(\sigma) \mathrm{d}_{\sigma}\right)^{1 / 2} \leq c h,
\end{aligned}
$$

where $c>0$ is a generic constant independent of $h$, and, due to the $L^{\infty}$ estimates on $u_{h}, \rho_{h}$, and $V_{h}$, it also holds

$$
\left|B-\int_{\Omega}\left(\log u_{h}-2 \log \rho_{h}+V_{h}\right) \rho_{h} \phi d x\right| \leq c h .
$$

Since $\varepsilon^{2} A-B=0$, the above computations imply (28).

Passing to the limit as $h \rightarrow 0$ in (27), (28), and (29) shows that the sequence $(\widehat{\rho}, \widehat{V}, \widehat{u})$ obtained in Lemma 9 is a weak solution to (4)-(8), which finishes the proof of Theorem 2. 


\section{NumericAl EXAMPLES}

In this section, we present some numerical simulations of a two-dimensional ballistic diode and a resonant tunneling diode (RTD). The ballistic GaAs $n^{+} n n^{+}$diode, whose geometry is shown in Figure 2, consists of a highly doped source and drain region (the doping density equals $10^{24} \mathrm{~m}^{-3}$ ) and a moderately doped channel (doping density of $5 \cdot 10^{21} \mathrm{~m}^{-3}$ ).

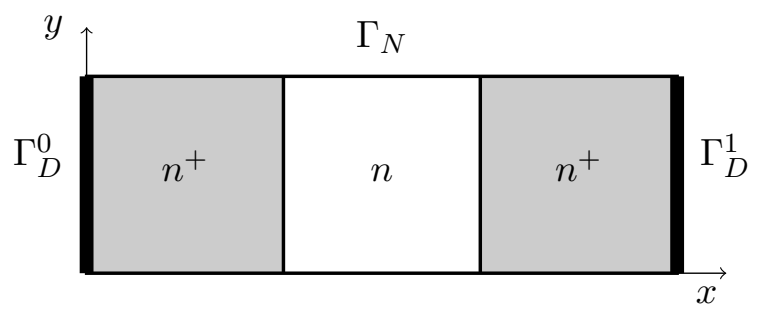

Figure 2. Geometry of the ballistic diode

For the scaled model this results in $\Omega=(0,1) \times(0,1 / 3), \Gamma_{D}=\Gamma_{D}^{0} \cup \Gamma_{D}^{1}(\{0\} \times(0,1 / 3)) \cup$ $(\{1\} \times(0,1 / 3))$, and the doping profile $C(x)$ is given by

$$
C(x, y)=\left\{\begin{array}{cl}
5 \cdot 10^{-3} & \text { if } 1 / 3<x<2 / 3 \\
1 & \text { else. }
\end{array}\right.
$$

The resonant tunneling diode has the same $n^{+} n n^{+}$structure as the ballistic diode but the channel is replaced by a quantum well sandwiched between two AlGaAs barriers. The double barrier structure is placed between two GaAs spacer layers, as shown in Figure 3 (a). We stress the fact that this structure is very simplified but captures the main device geometry; we refer to [31] for a more realistic geometry for a resonant tunneling transistor. Moreover, this section is intended to verify the properties of the numerical scheme rather than to simulate full-feature physical devices.

The physical effect of the barriers is a shift in the quasi-Fermi potential level, which is modeled by an additional step function $B(x, y)=B(x)$, depicted in Figure 3 (b), added to the electric potential. From a numerical point of view, we replace $V_{K}$ in (16) by $V_{K}-B_{K}$, where $B_{K}$ is the $L^{2}$ projection of $B$ on $K$. The step function $B$ is a piecewise constant function, which equals $\mathcal{B}$ (the barrier height) in the barriers and 0 else.

For the ballistic and the resonant tunneling diodes, the scaled boundary conditions are $n_{D}=1$ on $\Gamma_{D}, V_{D}=0$ on $\Gamma_{D}^{0}$ and $V_{D}=V_{\text {ext }}$ on $\Gamma_{D}^{1}$. With these values, it follows that $\rho_{D}=\sqrt{n_{D}}, u_{D}=n_{D} e^{-V_{D}}$ and $G_{D}=\log u_{D}-\log n_{D}$ in $\Omega$.

The devices are simulated using the quantum drift-diffusion equations as discretized in scheme (16)-(19). The first two equations (16) and (17) are numerically solved by Newton's method, whereas a fixed-point iteration is employed for the full scheme. The iteration procedure is coupled to a continuation in the applied voltage, i.e., the discrete system is solved for some applied voltage $V_{\text {ext }}+\triangle V$, using the solution for $V_{\text {ext }}$ as an initial guess, and $\triangle V$ is the voltage increment.

5.1. A test case. First, we consider a test case presented by Pinnau in [32]. We choose a scaled Debye length $\lambda^{2}=10^{-2}$ and a scaled biasing voltage $V_{\text {ext }}=-5$. The scaled barrier potential $\mathcal{B}$ is set to 0 for the ballistic diode and to 1 for the resonant tunneling diode. Figure 4 presents the electron density for different values of the scaled Planck constant $\varepsilon$, computed 


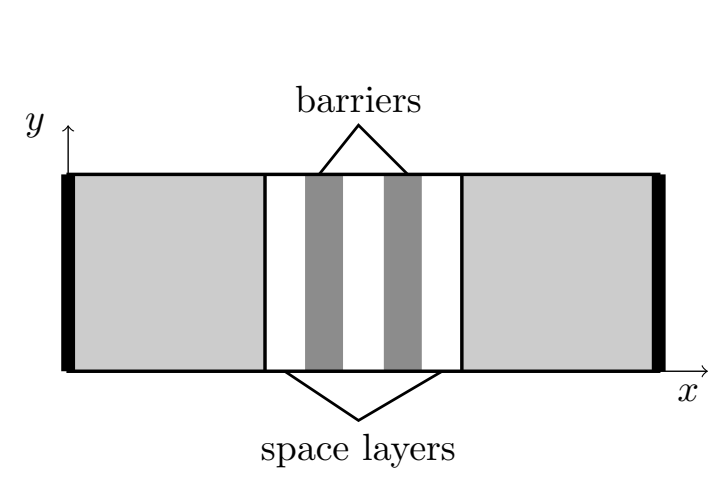

(a) Geometry

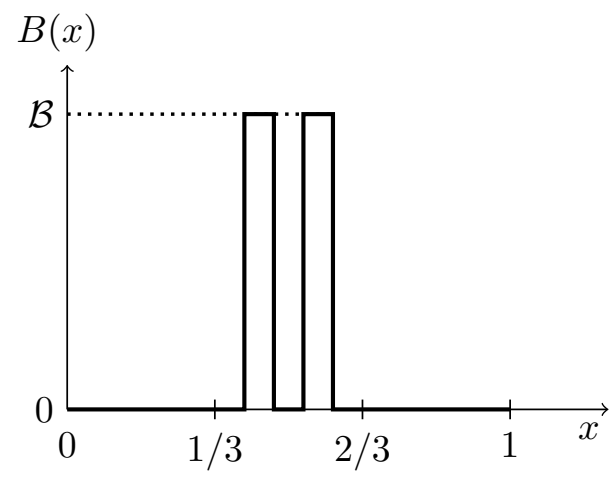

(b) Step function

FiguRE 3. Resonant tunneling diode

with the scheme (16)-(19) on a fine triangular mesh made of 10752 triangles. For $\varepsilon^{2}=10^{-5}$, we notice large gradients for the electron density close to the barriers. The profiles are similar to those obtained by Pinnau in [32] using a finite-element scheme in one space dimension.

Next, we wish to compute the convergence rates of the scheme. To this end, we compute the numerical solution to the scheme (16)-(19) on a sequence of rectangular meshes with only one cell in the $y$-direction. The coarsest grid is made of 30 rectangles and the sequence of meshes is obtained by dividing successively the rectangles by 2 . The finest grid is made of 7680 rectangles and the numerical solution on the finest grid is taken as reference solution for the computation of the $L^{2}$ error for the variables $n, V$ and $G$.

Figure 5 presents the error in $L^{2}$ norm between the approximate solution and the reference solution for the ballistic diode (left column) and the resonant tunneling diode (right column). The numerical results show a convergence rate of order one for all variables, even for rather small values of $\varepsilon$. However, for $\varepsilon^{2}=10^{-5}$, the first-order convergence rate is obtained for sufficiently small values of $h$ only.

5.2. Numerical simulation of a resonant tunneling diode. We consider now a GaAsAlGaAs double barrier structure. The geometry of this resonant tunneling diode is shown in Figure 3 (a) and the doping profile is the same as above. The physical parameters and the scaling are chosen as in [34]. More precisely, the length of the device is $L=750 \AA$ and the lattice temperature equals $T=77 \mathrm{~K}$. The physical constants are the reduced Planck constant $\hbar=1.05 \cdot 10^{-34} \mathrm{VAs}^{2}$, the Boltzmann constant $k_{B}=1.38 \cdot 10^{-23} \mathrm{VAsK}^{-1}$, the charge of electrons $q=1.6 \cdot 10^{-19}$ As and the permittivity of GaAs, $\epsilon=12.9 \epsilon_{0}=1.14 \cdot 10^{-10} \mathrm{AsV}^{-1} \mathrm{~m}^{-1}$. In the device, the effective electron mass is assumed to be constant $m=0.126 \cdot m_{0}=1.07 \cdot 10^{-31}$ $\mathrm{kg}$ and the mobility of electrons is $\mu=2.5 \mathrm{~m}^{2} \mathrm{~V}^{-1} \mathrm{~s}^{-1}$. Then the thermal voltage is defined by $U_{T}=k_{B} T / q=6.64 \cdot 10^{-3} \mathrm{~V}$ and the scaling yields

$$
\varepsilon^{2}=\frac{\hbar^{2}}{6 k_{B} T m L^{2}}=2.87 \cdot 10^{-3}, \quad \lambda^{2}=\frac{\epsilon U_{T}}{q C_{m} L^{2}}=8.41 \cdot 10^{-4} .
$$

Notice that the effective electron mass constant is chosen larger than the physical value for GaAs, $m=0.067 \cdot m_{0}$, in order to emphasize the negative differential resistance effect in the voltage-current characteristics (see below). 

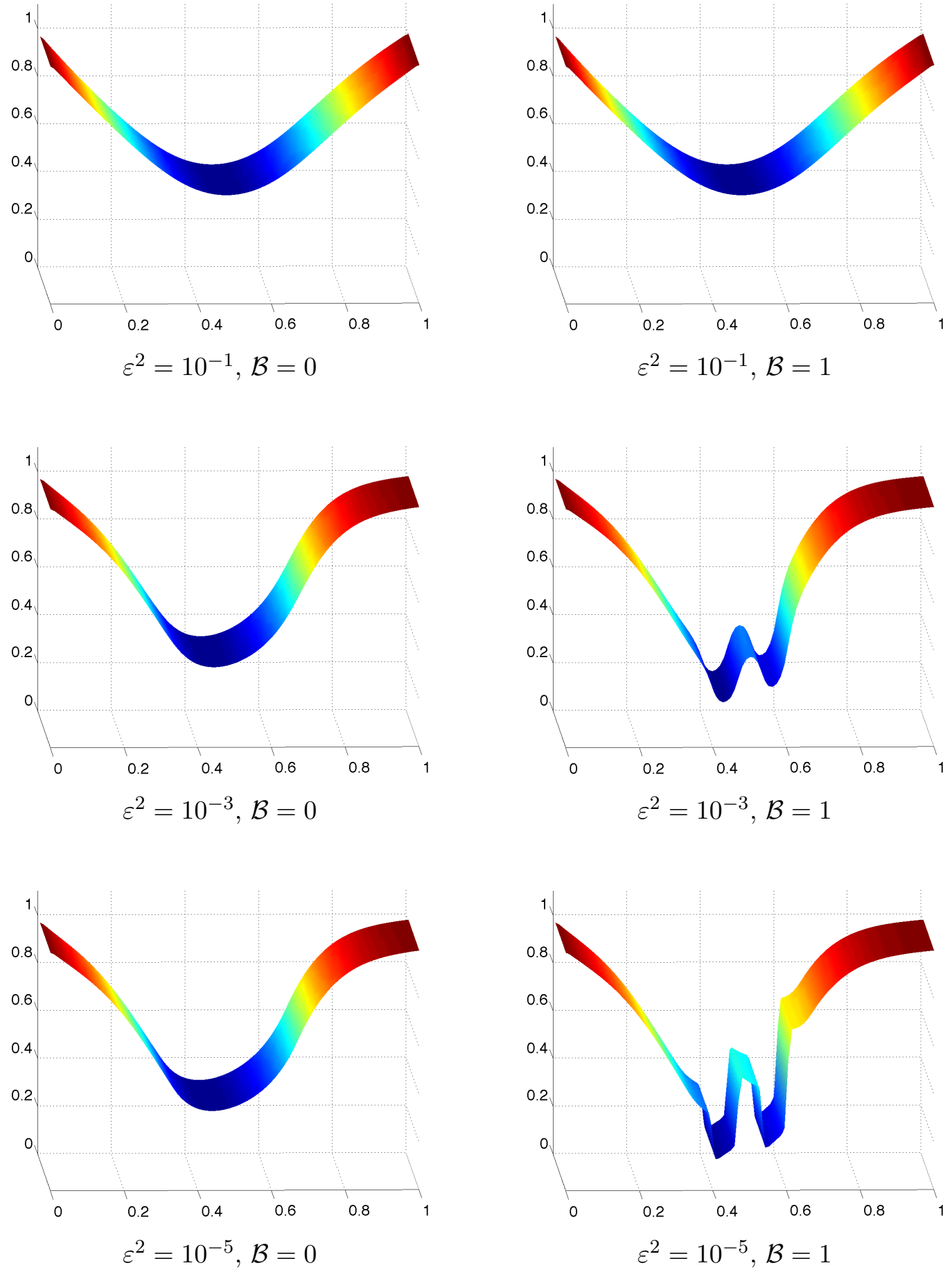

FiguRE 4. Electron densities in the diode (left: ballistic, right: RTD) for different values of $\varepsilon^{2}$. 

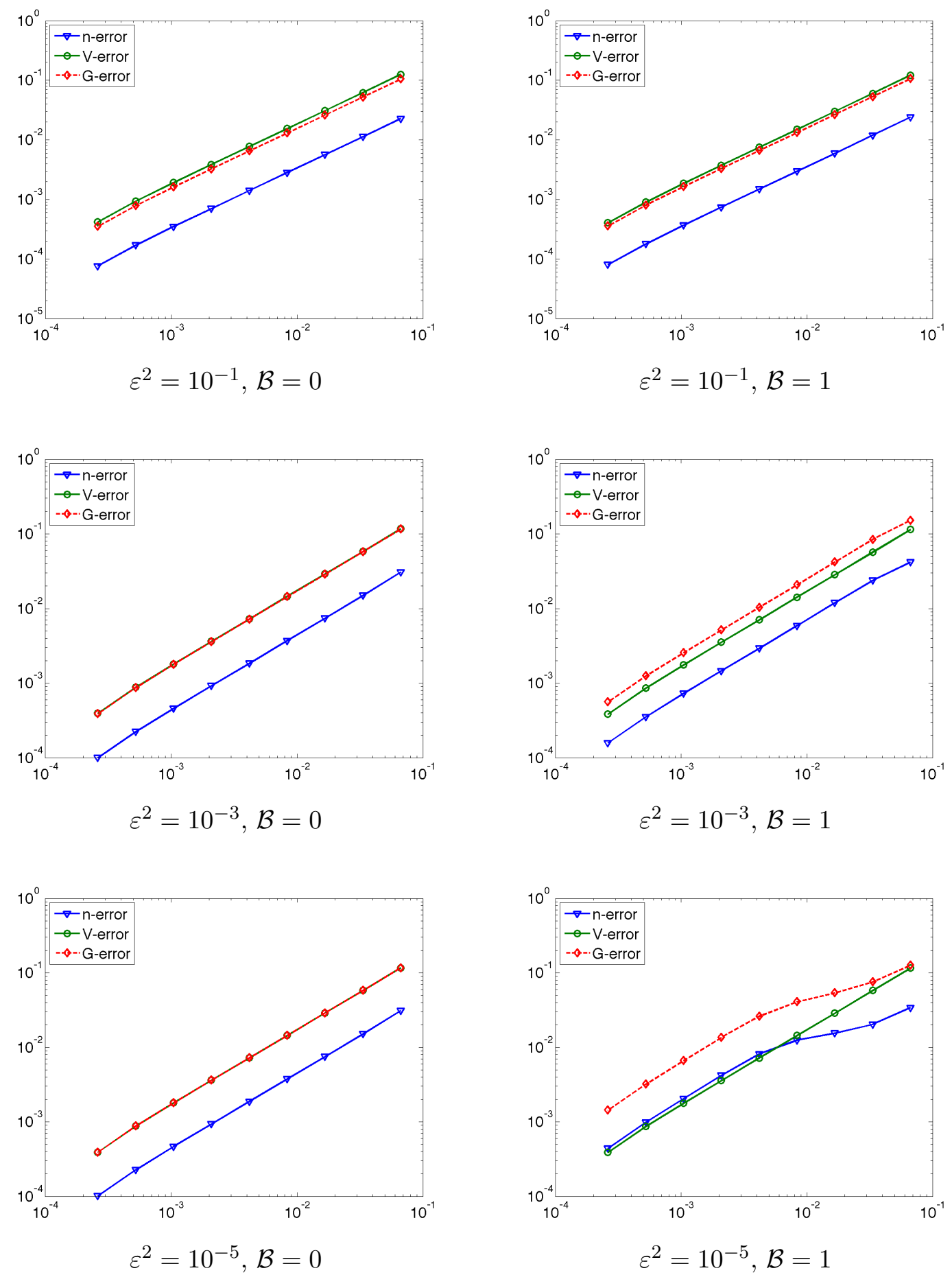

FIGURE 5. Error estimates in $L^{2}$ norm (left: ballistic, right: RTD) for different values of $\varepsilon^{2}$. 
First, we compute the thermal equilibrium (applied voltage $V_{\text {ext }}=0 \mathrm{~V}$ ). The electron density $n$ and the electric potential $V$ are shown in Figure 6 for different values of the barrier height: $\mathcal{B}=0 \mathrm{eV}$ (ballistic case), $\mathcal{B}=0.1 \mathrm{eV}$ and $\mathcal{B}=0.2 \mathrm{eV}$. Again we observe large gradients of the electron density close to the barriers.

Figure 7 shows the current-voltage characteristics when the barrier height is assumed to be $\mathcal{B}=0.3 \mathrm{eV}$. The peak is achieved at the potential $V_{\text {ext }}=-0.18 \mathrm{~V}$ and the valley at $V_{\text {ext }}=-0.245 \mathrm{~V}$. The nonmonotone behavior of the current-voltage curve is called negative differential resistance effect, which allows one to use these devices in ultra high-speed circuits. Finally, the electron densities at the peak and the valley are presented in Figure 8.

\section{Conclusions}

In this paper, we have analyzed and numerically tested a finite-volume scheme for the multidimensional quantum drift-diffusion equations with physically motivated boundary conditions. The existence of solutions to the discrete scheme was proved employing the Brouwer fixed-point theorem and the minimization of the discrete energy functional. As a by-product of the minimization procedure, we obtained discrete $H^{1}$ a priori estimates, which allowed us to prove the convergence of the discrete solutions to a solution to the continuous model. Numerical tests have shown that the convergence rate is of order one. Interestingly, the convergence rates seem to be uniform in the scaled Planck constant. A finite-element scheme with such a property was derived by Pinnau for the one-dimensional model, see [32]. Furthermore, we have simulated a simple two-dimensional resonant tunneling diode with a slightly enlarged effective mass constant, showing negative differential resistance. Our results show the good performance and stability of the finite-volume scheme.

\section{REFERENCES}

[1] M. Ancona. Diffusion-drift modeling of strong inversion layers. COMPEL 6 (1987), 11-18.

[2] M. Ancona. Finite-difference schemes for the density-gradient equations. J. Comput. Electr. 1 (2002), 435-443.

[3] M. Ancona, Z. Yu, R. Dutton, P. Vande Voorde, M. Cao, and V. Vook. Density-gradient analysis of MOS tunneling. IEEE Trans. Electron Devices 47 (2000), 2310-2319.

[4] A. Asenov, S. Kaya, J. Davies, and S. Saini. Oxide thickness variation induced threshold voltage fluctuations in decanano MOSFET: A 3d density gradient simulation study. Superlattices Microstruct. 28 (2000), 507-515.

[5] N. Ben Abdallah and A. Unterreiter. On the stationary quantum drift diffusion model. Z. Angew. Math. Phys. 49 (1998), 251-275.

[6] C. Chainais-Hillairet and F. Filbet. Asymptotic behavior of a finite volume scheme for the transient drift-diffusion model. IMA J. Numer. Anal. 27 (2007), 689-716.

[7] C. Chainais-Hillairet, J.-G. Liu and Y.-J. Peng. Finite volume scheme for multi-dimensional drift-diffusion equations and convergence analysis. M2AN 37 (2003), 319-338.

[8] Y. Coudière, T. Gallouët, and R. Herbin. Discrete Sobolev inequalities and $L^{p}$ error estimates for finite volume solutions of convection diffusion equations. Math. Mod. Numer. Anal. 35 (2001), 767-778.

[9] S. Datta. Nanoscale device modeling: the Green's function method. Superlattices Microstruct. 28 (2000), 253-278.

[10] P. Degond, F. Méhats, and C. Ringhofer. Quantum energy-transport and drift-diffusion models. J. Stat. Phys. 118 (2005), 625-665.

[11] R. Eymard and T. Gallouët. H-convergence and numerical schemes for elliptic problems. SIAM J. Numer. Anal. 41 (2003), 539-562.

[12] R. Eymard, T. Gallouët, and R. Herbin. Finite Volume Methods. In: Handbook of Numerical Analysis. Vol. 7, pp. 713-1020. North-Holland, Amsterdem, 2000. 

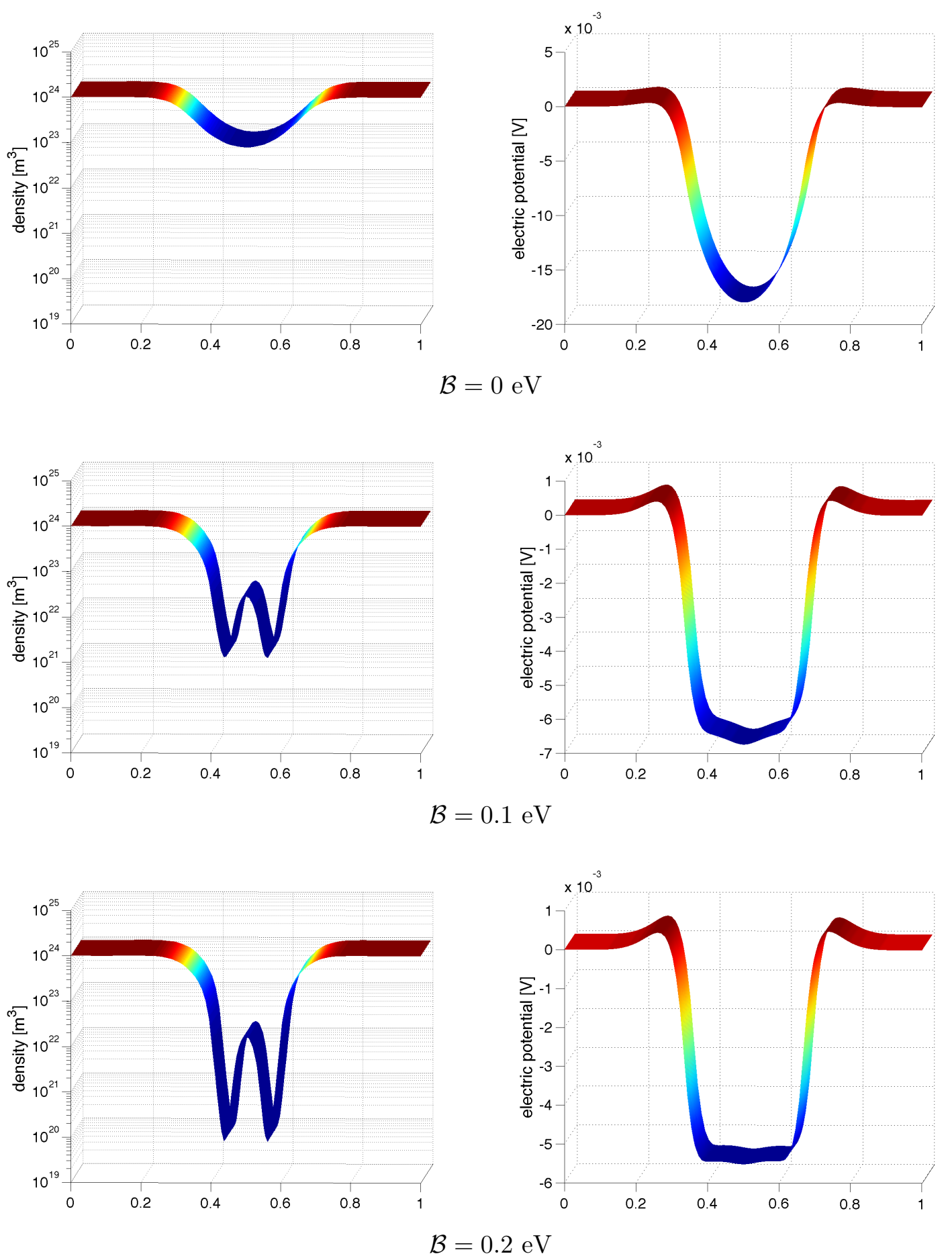

Figure 6. Equilibrium densities (left) and electric potential (right) for different values of the barrier height. 


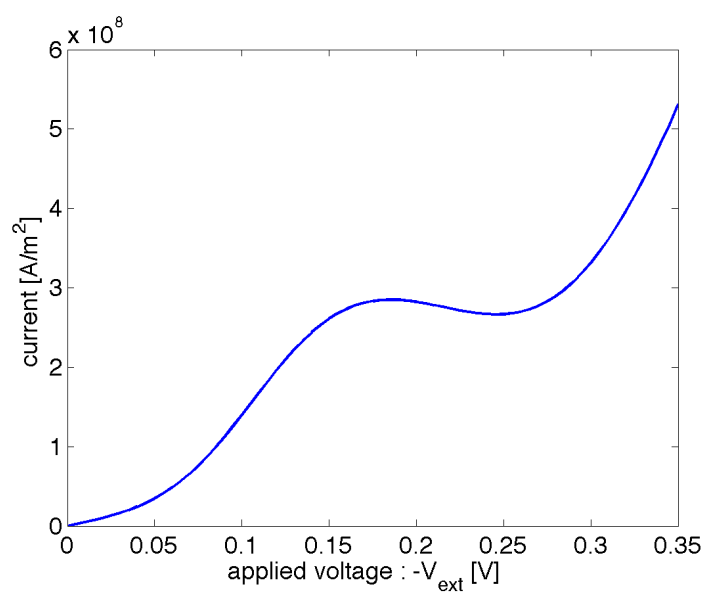

FiguRE 7. Current-voltage characteristics for a barrier height $\mathcal{B}=0.3 \mathrm{eV}$.
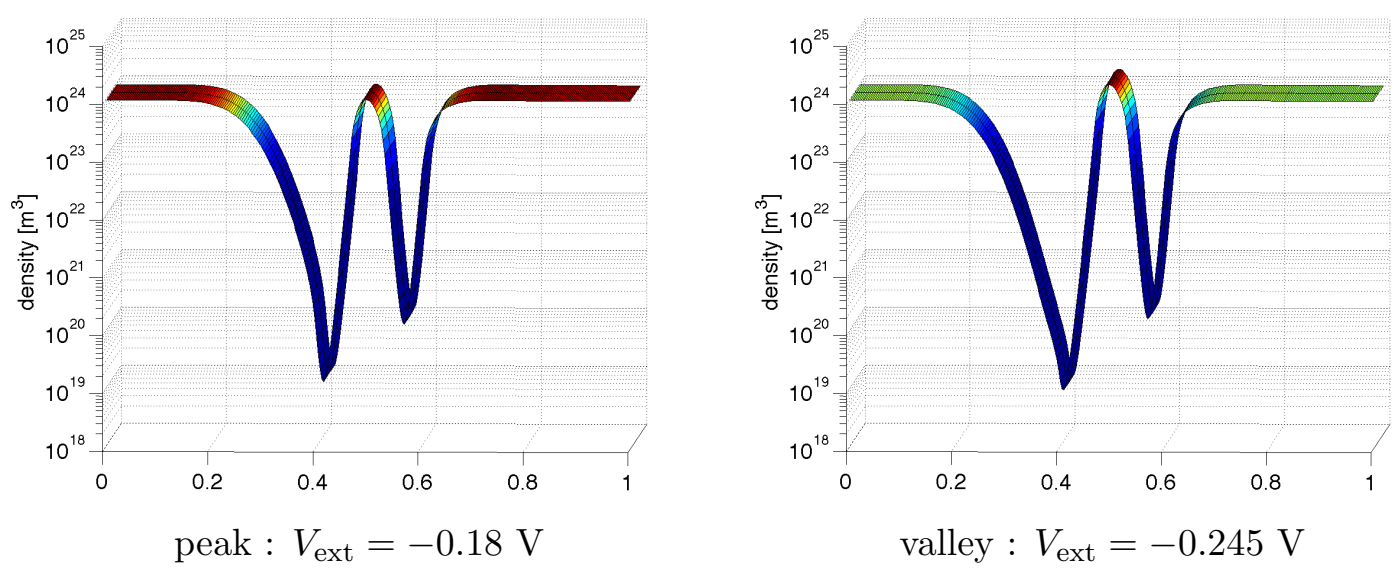

Figure 8. Densities at the peak (left) and the valley ( right)

[13] R. Eymard, T. Gallouët, and R. Herbin. Discretisation of heterogeneous and anisotropic diffusion problems on general nonconforming meshes. SUSHI: a scheme using stabilisation and hybrid interfaces. To appear in IMA J. Numer. Anal. (2009).

[14] C. de Falco, E. Gatti, A. Lacaita, and R. Sacco. Quantum-corrected drift-diffusion models for transport in semiconductor devices. J. Comput. Phys. 204 (2005), 533-561.

[15] D. Ferry and J.-R. Zhou. Form of the quantum potential for use in hydrodynamic equations for semiconductor device modeling. Phys. Rev. B 48 (1993), 7944-7950.

[16] W. Frensley. Wigner-function model of a resonant-tunneling semiconductor device. Phys. Rev. B 36 (1987), 1570-1580.

[17] T. Gallouët. Nonlinear methods for linear equations. In: Proceedings of TAMTAM, Tipaza, Algeria (2007).

[18] C. Gardner. The quantum hydrodynamic model for semiconductor devices. SIAM J. Appl. Math. 54 (1994), 409-427.

[19] U. Gianazza, G. Savaré, and G. Toscani. The Wasserstein gradient flow of the Fisher information and the quantum drift-diffusion equation. To appear in Arch. Ration. Mech. Anal., 2009.

[20] A. Jüngel. A steady-state quantum Euler-Poisson system for semiconductors. Commun. Math. Phys. 194 (1998), 463-479. 
[21] A. Jüngel. Transport Equations for Semiconductors. Lecture Notes in Physics 773. Springer, Berlin, 2009, to appear.

[22] A. Jüngel, H.-L. Li, and A. Matsumura. The relaxation-time limit in the quantum hydrodynamic equations for semiconductors. J. Diff. Eqs. 225 (2006), 440-464.

[23] A. Jüngel and D. Matthes. The Derrida-Lebowitz-Speer-Spohn equation: existence, non-uniqueness, and decay rates of the solutions. SIAM J. Math. Anal. 39 (2008), 1996-2015.

[24] A. Jüngel and R. Pinnau. A positivity-preserving numerical scheme for a nonlinear fourth order parabolic equation. SIAM J. Numer. Anal. 39 (2001), 385-406.

[25] A. Jüngel and I. Violet. The quasineutral limit in the quantum drift-diffusion equations. Asympt. Anal. 53 (2007), 139-157.

[26] S. Laux and F. Stern. Electron states in narrow gate-induced channels in Si. Appl. Phys. Lett. 49 (1986), 91-93.

[27] P. Markowich. The Stationary Semiconductor Device Equations. Springer, Vienna, 1986.

[28] C. Moglestue. Self-consistent calculation of electron and hole inversion charges at silicon-silicon dioxide interfaces. J. Appl. Phys. 59 (1986), 3175-3183.

[29] S. Odanaka. Multidimensional discretization of the stationary quantum drift-diffusion model for ultrasmall MOSFET structures. IEEE Trans. Computer-Aided Design Integr. Circ. Sys. 23 (2004), 837-842.

[30] F. Pacard and A. Unterreiter. A variational analysis of the thermal equilibrium state of charged quantum fluids. Commun. Part. Diff. Eqs. 20 (1995), 885-900.

[31] W. Peatman, E. Brown, M. Rooks, P. Maki, W. Grimm, and M. Shur. Novel resonant tunneling transistor with high transconductance at room temperature. IEEE Electron Device Letters 15 (1994), 236-238.

[32] R. Pinnau. Uniform convergence of the exponentially fitted scheme for the quantum drift-diffusion model. SIAM J. Numer. Anal. 42 (2004), 1648-1668.

[33] R. Pinnau and J. Ruiz V. Convergent finite element discretizations of the density gradient equation for quantum semiconductors. J. Comput. Appl. Math. 223 (2009), 790-800.

[34] R. Pinnau and A. Unterreiter. The stationary current-voltage characteristics of the quantum drift-diffusion model. SIAM J. Numer. Anal. 37 (1999), 211-245.

[35] C. Rafferty, B. Biegel, Z. Yu, M. Ancona, J. Bude, and R. Dutton. Multi-dimensional quantum effect simulation using a density-gradient model and script-level programming techniques. In: Proc. SISPAD, Sept. 1998 (1998), 137-140.

[36] G. Stampacchia. Le problème de Dirichlet pour les équations élliptiques du second ordre à coéfficients discontinus. Annales de l'Institut Fourier 15 (1965), 189-257.

[37] A. Wettstein, A. Schenk, and W. Fichtner. Quantum device-simulation with the density-gradient model on unstructured grids. IEEE Trans. Electron Devices 48 (2001), 279-284.

Laboratoire de Mathématiques, CNRS UMR 6620, Université Blaise Pascal (Clermont-FerRAND 2), 63177 Aubière, France; E-mail: Claire.Chainais@MATh.univ-BPClermont.Fr

Laboratoire de Mathématiques, CNRS UMR 5127, Université de Savoie, 73376 Le Bourget-DuLAC, FranCE; E-MAIL: GISCLON@UNIV-SAVOIE.FR

Institute for Analysis and Scientific Computing, Vienna University of Technology, Wiedner Hauptstr. 8-10, 1040 Wien, Austria; E-Mail: Juengel@Anum.tuwien.AC.AT 